\title{
The H-mode pedestal structure and its role on confinement in JET with a carbon and metal wall
}

\author{
M.J. Leyland ${ }^{1}$, M.N.A. Beurskens ${ }^{2}$, L. Frassinetti ${ }^{3}$, C. Giroud ${ }^{2}$, S. Saarelma ${ }^{2}$, P.B. \\ Snyder $^{4}$, J. Flanagan ${ }^{2}$, S. Jachmich ${ }^{5}$, M. Kempenaars ${ }^{2}$, P. Lomas ${ }^{2}$, G. Maddison ${ }^{2}$, \\ R. Neu ${ }^{6,7}$, I. Nunes ${ }^{8}$, K.J. Gibson ${ }^{1}$ and JET-EFDA Collaborators ${ }^{\dagger}$ \\ JET-EFDA, Culham Science Centre, Abingdon, OX14 3DB, UK \\ ${ }^{1}$ York Plasma Institute, Department of Physics, University of York, Heslington, York, YO1O 5DD, UK. \\ ${ }^{2}$ EURATOM /CCFE Fusion Association, Culham Science Centre, Abingdon, OX14 3DB, UK \\ ${ }^{3}$ Division of Fusion Plasma Physics, Association EURATOM-VR, KTH, SE-10044 Stockholm, Sweden \\ ${ }^{4}$ General Atomics, PO Box 85608, San Diego, California 92186-5608, USA \\ ${ }^{5}$ Laboratory for Plasma Physics, ERM/KMS, EURATOM-Association “Belgian State”, Belgium \\ ${ }^{6}$ Max-Planck-Institut für Plasmaphysik, D-85748 Garching, Germany \\ ${ }^{7}$ Technische Universtät München, D-85748 Garching, Germany \\ ${ }^{8}$ IPFN, EURATOM-IST Associação, 1096 Lisbon, Portugal. \\ ${ }^{\dagger}$ See the Appendix of F. Romanelli et al., Proc. of the 24th IAEA Fusion Energy Conf. 2012, San Diego, \\ US
}

Email: Matthew.Leyland@ccfe.ac.uk

\section{Abstract}

We present the pedestal structure, as determined from the high resolution Thomson scattering (HRTS) measurements, for a database of low and high triangularity $(\delta \approx$ 0.22-0.39) 2.5MA, Type I ELMy H-mode JET plasmas after the installation of the new ITER-like Wall (JET-ILW). The database explores the effect of increasing deuterium fuelling and nitrogen seeding with a view to explain the observed changes in performance (edge and global). The low triangularity JET-ILW plasmas show no significant change in performance and pedestal structure with increasing gas dosing. These results are in good agreement with EPED1 predictions. At high triangularity, for pure deuterium fuelled JET-ILW plasmas, there is a 20-30\% reduction in global performance and pressure pedestal height in comparison to JET-C plasmas. This reduction in performance is primarily due to a degradation of the temperature pedestal height. The global performance and pressure pedestal height of JET-ILW plasmas can be partially recovered to that of JET-C plasmas with additional nitrogen seeding [Giroud 2013]. This observed improvement in performance is predominately due to a significant increase in density pedestal height as well as a small increase in the temperature pedestal height. A key result with increasing deuterium fuelling for JETILW plasmas is there is no improvement in pressure pedestal height however the pedestal still widens which is inconsistent with the $\Delta=0.076 \sqrt{ } \beta_{\text {pol,ped }}$ scaling. Furthermore, a key result with increasing nitrogen seeding is the pressure pedestal widening is due to an increase in the temperature pedestal width whilst the density pedestal shows no clear trend. The comparison of EPED1 predictions with the measurements at high triangularity is complex as, for example, for pure deuterium fuelled plasmas there is very good agreement for the pedestal height but not the width. In addition, current EPED1 runs under-predict the pedestal height and width at high nitrogen seeding for JET-ILW plasmas however further work is required to determine the significance of these deviations. Understanding these deviations is essential as provides an insight to the physical mechanisms governing the pedestal structure and edge performance. 


\section{Introduction}

In H-mode (high confinement mode) operation in Tokamak devices, there is a steep pressure gradient at the plasma edge called the pedestal. The pedestal width and gradient both determine the achievable height. It is desirable to maximise the pedestal height as it is strongly linked to the plasma core performance [Ryter 2001, IPB-C2 2007]. However, a steeper and broader pedestal (a higher pedestal) is more susceptible to limiting instabilities. Common instabilities in the pedestal region are Edge Localised Modes (ELMs) as characterised by a periodic collapse of the pedestal due to reaching a critical width, gradient and height thought to be associated with crossing the Peeling Ballooning (PB) stability boundary [Connor 1998]. The challenge when operating with ELMs is mitigating the large transient heat fluxes on plasma facing components. To achieve the desired global performance on ITER, while limiting the steady state and transient divertor heat loads, it is important to exploit current operational devices, such as JET, to further understand the physical mechanisms governing the pedestal and the impact on global ELMy H-mode performance.

The ITER-Like-Wall (ILW) was installed on the JET Tokamak in 2010/11 with the primary aim of demonstrating a reduction in fuel retention [Matthews 2011, Philipps 2010]. The material composition of the plasma facing components is Beryllium for the main chamber and Tungsten for the high heat flux regions, as foreseen for ITER [Pitts 2009]. Measurements from recent JET campaigns with the ILW offer an invaluable opportunity to investigate how the pedestal structure changes with the presence of a metallic wall and its role on confinement. This paper presents a database consisting of deuterium fuelled and nitrogen seeded Type I ELMy H-mode plasmas on JET with the ILW (JET-ILW) [Maddison 2011, Giroud-IAEA-2012, Giroud 2013, Beurskens 2013, Beurskens 2014, Maddison 2014] with the focus on quantifying the pedestal structure. The pedestal width, gradient and height is determined by fitting a modified hyperbolic tangent (mtanh) function [Groebner 1998] to JET High Resolution Thomson Scattering (HRTS) radial profiles of electron temperature and density [Pasqualotto 2004, Frassinetti 2012, Scannell 2011].

Three high triangularity Type I ELMy H-mode plasmas on JET with the Carbon Fibre Composite wall (JET-C), presented in [Leyland 2013], are used within this study as reference plasmas before the installation of the Be/W wall. These three JET-C plasmas have high resolution HRTS measurements at the plasma edge sufficient to quantify the pedestal structure. To summarise, [Leyland 2013] quantifies the role of pedestal structure on performance across a deuterium fuelling scan. The plasma performance was not only maintained but even improved with increased fuelling up to a density pedestal normalised to the Greenwald density $\left(\mathrm{n}_{\mathrm{e}, \mathrm{ped}} / \mathrm{n}_{\mathrm{GW}}\right) \approx 1$. This improvement in performance was attributed to an increase in pedestal stored energy which coincided with a transition from pure Type I ELMs to mixed Type I/II ELMs [Saibene 2002, Saibene 2005, Giroud 2012]. A key result was that the pre-ELM electron temperature and density pedestal width increased from low to high deuterium fuelling.

Results from the 2012 JET-ILW campaign show the high triangularity Type I ELMy $\mathrm{H}$-mode baseline plasmas exhibit an approximate $20-30 \%$ reduction in performance in comparison to JET-C plasmas. This can be attributed to a degraded pressure pedestal 
height [Giroud 2013, Maddison 2014, Joffrin 2014]. However, with nitrogen seeding the pressure pedestal height and consequently global performance for JET-ILW plasmas can be partially recovered [Giroud-IAEA-2012, Giroud 2013, Maddison 2014]. These changes in performance coincide with a variation of the peak pedestal gradient and the pedestal width. The reduction in the pressure pedestal height after the installation of the $\mathrm{Be} / \mathrm{W}$ wall is due to a reduction in temperature pedestal height. The recovery in pressure pedestal height with increasing nitrogen seeding is due to a significant increase in density pedestal height as well as an increase in temperature pedestal height.

A multi-machine review [Beurskens 2013] of three possible mechanisms which could account for the changes in performance observed on JET and AUG concluded that the improvement in performance is not due to an improvement in core confinement nor can it be accounted for due to ion dilution. Instead it is most likely the change in pedestal structure results in the improvement in global performance. More specifically, with increasing Nitrogen seeding for JET-ILW plasmas the pedestal widens and the peak gradient increases both contributing towards an increase pedestal pressure. Furthermore [Beurskens 2013] compares the measurements to preliminary results from the predictive pedestal structure model, EPED.

In [Beurskens 2013] only the pressure pedestal structure is discussed for high triangularity JET plasmas. This paper extends the JET-ILW pedestal analysis by considering a wider dataset of fuelling and seeding plasmas, incorporating more high triangularity JET-ILW plasmas as well as including low triangularity JET-ILW plasmas. In addition, the relevant contributions of the electron temperature and density pedestal are quantified along with a comparison of measurements with the most recent EPED model predictions.

The paper is organised as follows: Section 2 provides a description of the database and the plasmas presented in this study, Section 3 gives an overview of the JET measurements discussed throughout the study along with a detailed introduction to the EPED model; Section 4 presents the performance and pedestal structure of the vertical and horizontal target low triangularity JET-ILW plasmas; Section 5 presents the performance and pressure pedestal structure of the high triangularity JET-C and JETILW plasmas as well as a wider database comparison with EPED model predictions; and Section 6 provides a summary and discusses the conclusions. 


\section{Description of database}

The baseline Type I ELMy H-mode JET plasmas discussed in this study have a magnetic field and plasma current of $2.7 \mathrm{~T} / 2.5 \mathrm{MA}$ where $\mathrm{q}_{95} \approx 3.5$. The input power is $\approx 14-17 \mathrm{MW}$ corresponding to $\beta_{\mathrm{N}} \approx 1.2-1.5$. The triangularity $(\delta)$ ranges between $\approx$ $0.22-0.42$, see Table 1 . These plasmas are predominantly from the JET fuelling and seeding experiment before and after the installation of the Be/W ILW [Maddison 2011, Giroud 2012, Giroud-IAEA-2012, Giroud 2013, Maddison 2014]. The primary aim of this experiment is to develop a radiative scenario with the introduction of an impurity to mitigate divertor heat loads. As part of the experimental procedure there exists deuterium fuelling and nitrogen seeding scans suitable for investigating the change in pedestal structure. This study also incorporates deuterium fuelled plasmas from the JET baseline scenario development experiments [Joffrin 2014].

Table 1. Summary of key parameters defining the plasma scenario for all JET pulses considered in this study. The triangularity $\delta$ is the average of the upper and lower triangularity. $I_{p}$ is the plasmas current. $B_{t}$ is the toroidal magnetic field strength. $P_{N B I}$ is the input power from neutral beam injection. $D_{2} \Gamma_{e l}$ is the range of deuterium fuelling for the particular pulses included in this study and similarly $N_{2} \Gamma_{e l}$ is the range nitrogen fuelling. $n_{e, p e d} / n_{G W}$ is the density pedestal height normalised to the Greenwald density.

\begin{tabular}{ccccc}
\hline & $\begin{array}{c}\text { High } \delta \\
\text { JET-C } \\
\text { horiz. target }\end{array}$ & $\begin{array}{c}\text { High } \delta \\
\text { JET-ILW } \\
\text { horiz. target }\end{array}$ & $\begin{array}{c}\text { Low } \delta \\
\text { JET-ILW } \\
\text { horiz. target }\end{array}$ & $\begin{array}{c}\text { Low } \delta \\
\text { JET-ILW } \\
\text { vert. target }\end{array}$ \\
\hline $\begin{array}{c}\text { No. } \\
\text { Pulses }\end{array}$ & 14 & 60 & 5 & 4 \\
$\delta$ & 0.42 & 0.38 & 0.27 & 0.22 \\
$\mathrm{I}_{\mathrm{p}} / \mathrm{B}_{\mathrm{t}}$ & $2.5 \mathrm{MA} / 2.6-2.7 \mathrm{~T}$ & $2.5 \mathrm{MA} / 2.6-2.7 \mathrm{~T}$ & $2.5 \mathrm{MA} / 2.6-2.7 \mathrm{~T}$ & $2.5 \mathrm{MA} / 2.8 \mathrm{~T}$ \\
$\mathrm{P}_{\mathrm{NBI}}$ & $14-15$ & $15-17$ & $15-17$ & 16 \\
$(\mathrm{MW})$ & & & & $0.6-4.3$ \\
$\mathrm{D}_{2} \Gamma_{\mathrm{el}}$ & $0.2-2.6$ & $0.6-3.0$ & - & - \\
$\left(\mathrm{x} 10^{22} \mathrm{el} / \mathrm{s}\right)$ & & & & \\
$\mathrm{N}_{2} \Gamma_{\mathrm{el}}$ & - & $0.0-3.8$ & $0.0-2.5$ & \\
$\left(\mathrm{x} 10^{22} \mathrm{el} / \mathrm{s}\right)$ & & & & $0.59-0.70$ \\
$\mathrm{n}_{\mathrm{e}, \mathrm{ped}} / \mathrm{n}_{\mathrm{GW}}$ & $0.75-1.05$ & $0.67-1.06$ & 0.74 \\
\hline
\end{tabular}

The 83 plasmas selected for this study can be categorised into four groups: high triangularity JET-C plasmas $(\delta \approx 0.42)$, high triangularity JET-ILW plasmas $(\delta \approx$ $0.38)$, low triangularity horizontal target JET-ILW plasmas $(\delta \approx 0.27)$ and low triangularity vertical target JET-ILW plasmas $(\delta \approx 0.22)$. Figure $1($ a) demonstrates the EFIT magnetic equilibrium for a high triangularity JET-C (JPN: 79503) and an equivalent JET-ILW (JPN: 82585) plasma. These plasmas are similar apart from a small change in upper triangularity (JET-C $\delta_{\mathrm{UP}} \approx 0.44$, JET-ILW $\delta_{\mathrm{UP}} \approx 0.39$ ) to minimise the interaction with the upper inner-wall structure [Giroud 2012, Joffrin 2014]. Figure 1(b) and 1(c) demonstrate there is a more significant difference between a low triangularity horizontal (JPN: 83177) and vertical (JPN: 83491) target plasma when comparing the magnetic equilibrium reconstruction. The outer strike point for all the high triangularity plasmas (JET-C and JET-ILW) is located on a Horizontal Target (HT); a horizontal tile mounted centrally at the bottom of the divertor, see 
Figure 1(c). As well as the low triangularity HT JET-ILW plasmas, also considered are low triangularity plasmas where the strike point is positioned on a Vertical Target (VT); a vertically mounted tile on the outboard side of the JET divertor, see Figure 1(c).

At high triangularity the deuterium fuelling ranges from $\approx 0.2$ to $3.8 \times 10^{22} \mathrm{el} / \mathrm{s}$ and similarly the nitrogen seeding ranges from $\approx 0.0-3.8 \times 10^{22} \mathrm{el} / \mathrm{s}$. The nitrogen seeded plasmas are also fuelled with deuterium ranging from $\approx 0.8$ to $2.9 \times 10^{22} \mathrm{el} / \mathrm{s}$. The plasmas which make up the two nitrogen scans presented in this study have a similar fixed level of deuterium fuelling of $\approx 0.8$ and $1.2 \times 10^{22} \mathrm{el} / \mathrm{s}$. For these plasmas the electron pedestal density normalised to the Greenwald density $\left(\mathrm{n}_{\mathrm{e}, \mathrm{ped}} / \mathrm{n}_{\mathrm{GW}}\right)$ ranges from $\approx 0.67$ to 1.06 for JET-C and JET-ILW plasmas, see Table 1 . The plasmas which exhibit an improvement in performance with increased deuterium fuelling (JET-C) [Leyland 2013] and nitrogen seeding (JET-ILW) correspond to a high normalised density $\left(\mathrm{n}_{\mathrm{e}, \mathrm{ped}} / \mathrm{n}_{\mathrm{GW}}\right)$ above 1.0. At low triangularity over a similar range of fuelling and seeding the normalised pedestal density $\left(\mathrm{n}_{\mathrm{e}, \mathrm{ped}} / \mathrm{n}_{\mathrm{GW}}\right)$ ranges from $\approx 0.59$ to 0.74 for JET-ILW plasmas.

In the context of the variation in pedestal structure there is not a suitable: nitrogen seeding scan for high triangularity JET-C plasmas due to the lack of high resolution measurements at the time of the experiment; a deuterium fuelling scan for low triangularity horizontal target JET-ILW plasmas; and a nitrogen seeding scan for low triangularity vertical target JET-ILW plasmas, (see Table 1). There are nitrogen seeded JET-C plasmas [Maddison 2011, Giroud 2012], however the edge resolution of HRTS is insufficient for this study. The analysis performed for this study has demonstrated it is important to consider plasmas with the same current, magnetic field, neutral beam input power, gas fuelling configuration and ideally the same machine conditioning. Taking into account these factors results in clearer trends, however this is not always possible hence the limited low triangularity dataset. This study incorporates plasmas from only the first JET-ILW campaign however a more extensive dataset is provided by the most recent JET-ILW campaigns.

(a)

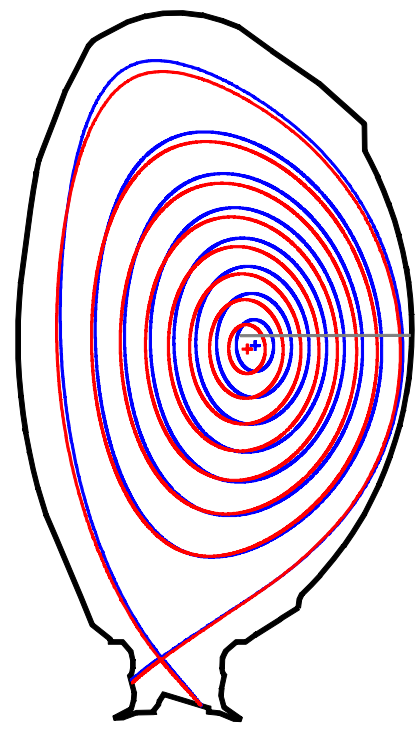

(b)

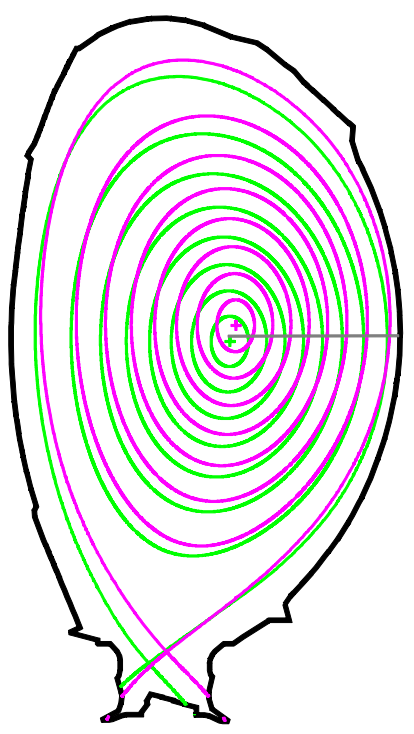


(c)

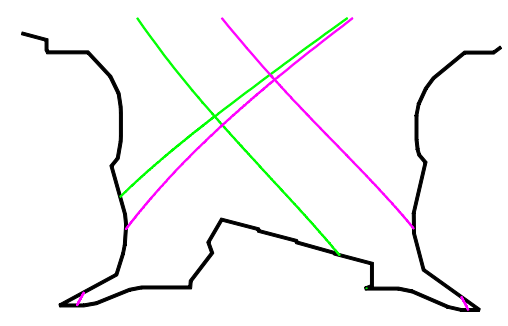

Figure 1. EFIT magnetic equilibrium reconstructions for (a) high triangularity JET-C plasma in blue $(J P N: 79503$ at $t=22.0$ s) and high triangularity JET-ILW plasma in red (JPN: 82585 at $t=15.8 s)$. (b) low triangularity horizontal target JET-ILW plasma in green (JPN: 83177 at 14.8s) with a low triangularity vertical target JETILW plasma in magenta (JPN: 83491 at 11.5s) (c) same as (b) with focus on the divertor region. The horizontal grey line shown on $(a)$ and $(b)$ indicates the location of the HRTS measurement. [Note: need to change colours] 


\section{Overview of JET measurements and EPED model}

The global performance is quantified by the confinement enhancement factor $\left(\mathrm{H}_{98}\right)$ and the stored thermal energy $\left(\mathrm{W}_{\mathrm{th}}\right) . \mathrm{H}_{98}$ is defined as the ratio of the measured energy confinement time for a specific pulse to the energy confinement time as determined from a scaling relation based upon an international multi machine database of $\mathrm{H}$-mode plasmas [IPB-C2-1999]. The stored thermal energy is calculated from the measured diamagnetic energy corrected for fast ions [ITER H-mode database working group 1994].

The pedestal stored energy $\left(\mathrm{W}_{\text {ped }}\right)$ is calculated by evaluating the volume integral of the pressure profile capped at the pressure pedestal top. The integral,

$$
W_{p e d}(J)=\frac{3}{2} \int\left(p_{i}+p_{e}\right) d V \approx \frac{3}{2} \int e\left(n_{i} T_{i}+n_{e} T_{e}\right) d V \approx \frac{1}{4} \int e\left(\frac{\left(Z_{I}+1\right)-Z_{e f f}}{Z_{I}}\right) n_{e} T_{e} d V
$$

is expressed in terms of the electron density $\left(n_{e}\right)$ in $m^{-3}$, electron temperature $\left(T_{e}\right)$ in $\mathrm{eV}$, the plasma volume $(\mathrm{V})$ in $\mathrm{m}^{3}$, the atomic charge of the dominate impurity $\left(\mathrm{Z}_{\mathrm{I}}\right)$, the average effective atomic charge $\left(\mathrm{Z}_{\mathrm{eff}}\right)$ and a constant $(\mathrm{e})$ the elementary unit of charge. The electron density and temperature profile utilised by Equation (1) are modified hyperbolic tangent fits to the pre-ELM HRTS measured profiles. Equation (1) assumes $T_{i} \sim T_{e}$ and uses the following relation between $n_{i}$ and $n_{e}$,

$$
n_{i}=\left(\frac{\left(Z_{I}+1\right)-Z_{e f f}}{Z_{I}}\right) n_{e} .
$$

For JET-C deuterium fuelled plasmas the dominant impurity is carbon $\left(Z_{I}=6\right)$. For JET-ILW deuterium fuelled plasmas the dominant impurity is beryllium $\left(Z_{I}=4\right)$ whereas for JET-ILW nitrogen seeded plasmas the dominant impurity is assumed to be nitrogen $\left(Z_{I}=7\right)$. Furthermore, it is important to note the average $Z_{\text {eff }}$ is incorporated into Equation (1) and a flat $Z_{\text {eff }}$ profile is assumed.

The poloidal pedestal normalised pressure $\left(\beta_{\text {pol,ped }}\right)$ is calculated using the expression given by [Beurskens 2011, Leyland 2013],

$$
\beta_{\text {pol,ped }}=\frac{p_{\text {ped }}}{B_{\text {pol }}^{2} / 2 \mu_{0}}=\frac{p_{\text {ped }}}{\left(\mu_{0} I_{P} / C\right)^{2} / 2 \mu_{0}}=\frac{2 C^{2} p_{\text {ped }}}{\mu_{0} I_{P}^{2}}
$$

expressed in terms of the total pressure pedestal height $\left(\mathrm{p}_{\mathrm{ped}}\right)$ in $\mathrm{Pa}$, the magnetic field strength (B) in $\mathrm{T}$, the plasma current $\left(\mathrm{I}_{\mathrm{p}}\right)$ in $\mathrm{A}$ and the plasma circumference $(\mathrm{C})$ in $\mathrm{m}$. The plasma circumference is determined from the magnetic equilibrium and the total pedestal pressure is calculated by again assuming $T_{i} \sim T_{e}$ and the relation between $n_{i}$ and $n_{e}$ given by Equation (2).

The pedestal structure is determined by fitting a modified hyperbolic tangent (mtanh) function to ELM synchronised JET High Resolution Thomson Scattering (HRTS) electron temperature and density profiles, see [Groebner 1998]. The HRTS system has a $20 \mathrm{~Hz}$ repetition rate resulting in $\approx 800$ profiles per pulse. Profiles are selected from the stationary ELMy H-mode phase of a pulse, typically $\approx 1.5-2.0 \mathrm{~s}(\approx 30-40$ profiles $)$. Furthermore, for pre-ELM fits the profiles are selected from the last 70-99\% of the ELM cycle. The size of the percentage window balances selecting a sufficient number 
of profiles for an accurate fit whilst only selecting profiles representative of the preELM state. The position of the profiles is corrected according to the position of the last closed flux surface as calculated from EFIT. This aligns the steep gradient region (the pedestal) accounting for either an error in the profile position or small scale fluctuations in plasma position during the ELM cycle. The JET pedestal fitting routine provides a so called classical deconvolved mtanh density fit and a weighted deconvolved temperature fit as determined using the HRTS instrument function [Frassinetti 2012, Scannell 2011]. The temperature fit takes into account the variation in density across the scattering volume particularly important in the steep gradient region of the profile. All the JET plasmas considered in this study have high resolution HRTS pedestal measurements where the FWHM of the instrument function is $\approx 11 \mathrm{~mm}$ [Frassinetti 2012].

Figure 2 shows an example of the deconvolved temperature and density mtanh fit for (a), (c) a pure deuterium fuelled high triangularity JET-ILW plasma (JET Pulse Number: 82585) and (b), (d) a nitrogen seeded high triangularity JET-ILW plasma (JET Pulse Number: 82814). The temperature and density pedestal widens from $\Delta_{\mathrm{Te}}$ $\approx 1.7$ to $2.5 \mathrm{~cm}$ and $\Delta_{\text {ne }} \approx 1.7$ to $2.2 \mathrm{~cm}$ respectively with the introduction of nitrogen. The temperature pedestal height remains constant at $\mathrm{T}_{\mathrm{e} \text {,ped }} \approx 0.7 \mathrm{keV}$ with the introduction of nitrogen whereas the density pedestal height significantly increases from $\mathrm{n}_{\mathrm{e} \text {,ped }} \approx 6.5$ to $10.0 \times 10^{19} \mathrm{~m}^{-3}$. Consequently the nitrogen seeded plasma has a higher electron pressure pedestal height as will be discussed in more detail in Section 5. As detailed above the total measured pressure pedestal height is calculated assuming the electron and ion temperature are equal and taking into account ion dilution (Equation 2).
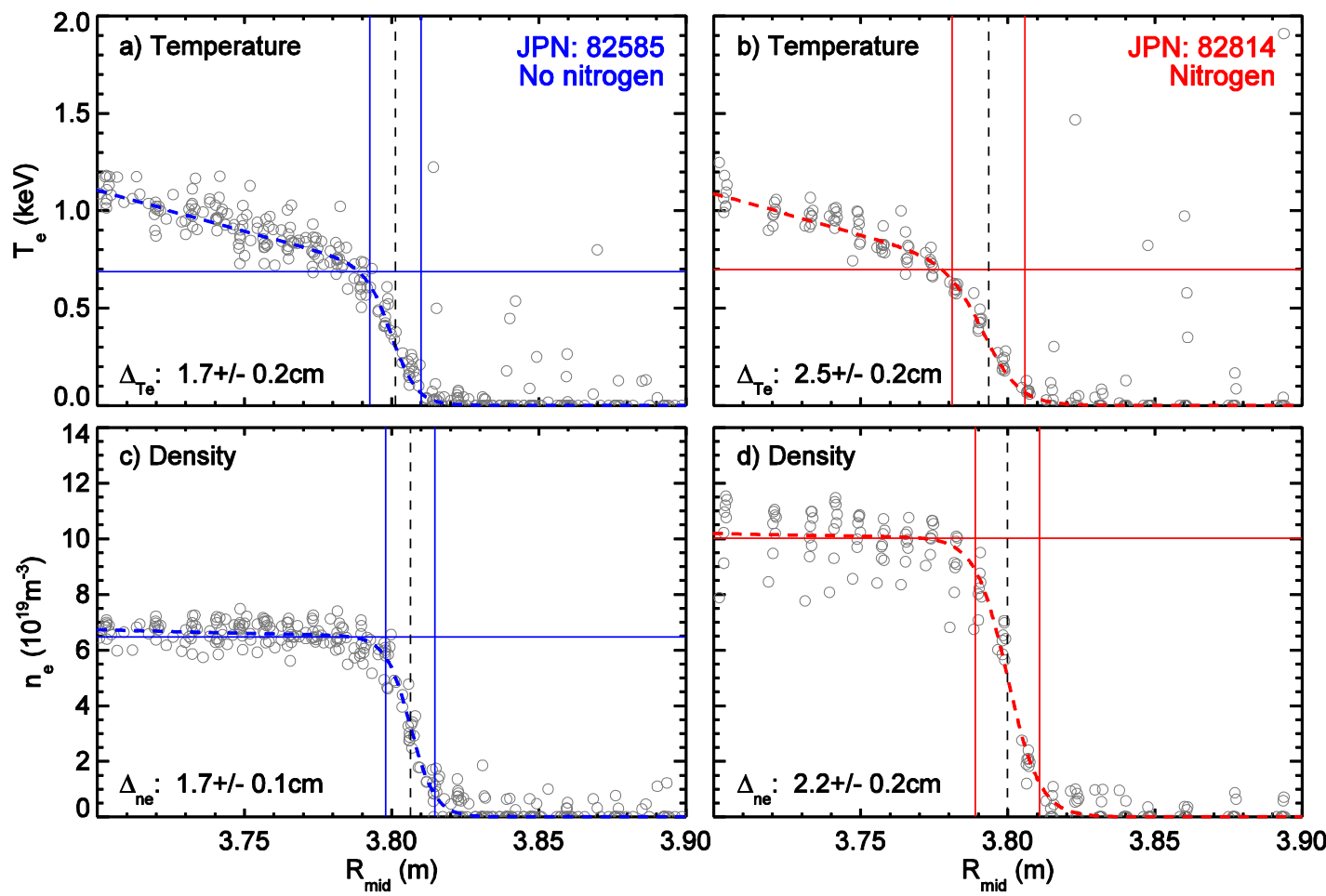

Figure 2. Deconvolved modified hyperbolic tangent fits to radial temperature and density HRTS profiles for (a), (c) a pure deuterium fuelled JET-ILW plasma (blue) 
where $D_{2} \Gamma_{e l}=1.1 \times 10^{22} \mathrm{el} / \mathrm{s}$ and $(b),(d)$ a nitrogen seeded JET-ILW plasma (red) where $D_{2} \Gamma_{e l}=1.3 \times 10^{22} \mathrm{el} / \mathrm{s}$ and $N_{2} \Gamma_{e l}=1.3 \times 10^{22} \mathrm{el} / \mathrm{s}$.

EPED, assumes that the pressure will rise indefinitely until the onset of two instabilities, peeling-ballooning modes and kinetic ballooning modes [Snyder 2009, Snyder 2011]. These two constraints are solved for the pedestal height and width. The model inputs are eight scalar parameters, $B_{t}(T)$ the toroidal magnetic field, $I_{p}(M A)$ the plasmas current, $\mathrm{R}(\mathrm{m})$ the major radius, a $(\mathrm{m})$ the minor radius, $\delta$ the triangularity, $\kappa$ the elongation, $\mathrm{n}_{\mathrm{e} \text {,ped }}\left(10^{19} \mathrm{~m}^{-3}\right)$ the pedestal density and $\beta_{\mathrm{N}, \text { global }}$ the global Troyon normalised pressure. There are two versions of the model, EPED1 [Snyder 2009] and EPED1.62 [Snyder 2011]. The predictions presented in this study are from EPED1 where the kinetic ballooning constraint is a square root relationship between pedestal width and normalised poloidal pedestal pressure $\left(\beta_{\mathrm{pol}, \mathrm{ped}}\right)$ with the constant of proportionality being 0.076 . The EPED1 runs for JET plasmas currently assume an up-down symmetric plasma shape.

Previous studies which discuss EPED1 predictions of the JET pedestal include [Beurskens-PPCF-2009, Beurskens 2011, Leyland 2013, Beurskens 2014]. The earlier multi machine comparisons (JET, DIII-D measurements [Beurskens-PPCF-2009] and JET, DIII-D, AUG measurements [Beurskens 2011]) concluded there is a good agreement between the predicted and measured pedestal height. The conclusion from more recent extensive comparisons, dedicated to JET-C [Leyland 2013] and JET-ILW [Beurskens 2014] measurements, is more complex as, for example, there are discrepancies between the predicted and measured pedestal height at high deuterium fuelling (JET-C) and high nitrogen seeding (JET-ILW). A possible explanation regarding the discrepancy for highly fuelled JET-C plasmas is due to EPED underpredicting the critical density as a consequence of measurement uncertainty on an additional input parameter to the model, $Z_{\text {eff }}$ [Leyland 2013]. The critical density marks the transition from peeling to ballooning limited plasmas.

Further to the eight traditional EPED inputs $\left(\mathrm{B}_{\mathrm{t}}, \mathrm{I}_{\mathrm{p}}, \mathrm{R}, \mathrm{a}, \delta, \kappa, \mathrm{n}_{\mathrm{e}, \mathrm{ped}}, \beta_{\mathrm{N}, \mathrm{global}}\right)$ the local pedestal effective atomic charge $\left(Z_{\text {eff }}\right)$ can also be included. This is a particularly important parameter in the context of this study as, for example, the change from a carbon to a metal wall and the variation of nitrogen seeding is incorporated into the EPED model by varying $Z_{\text {eff. }}$. However, quantifying the variation of the radial $Z_{\text {eff }}$ profile in the pedestal region due to impurity seeding is challenging on JET. There are non-local bremsstrahlung line-integral measurements of $Z_{\text {eff }}$ [Morgan 1985], which for JET-C plasmas, decrease from $\approx 2.0$ to 1.7 with increasing deuterium fuelling demonstrating the plasma becomes purer [Giroud 2012]. After the installation of the $\mathrm{Be} / \mathrm{W}$ ILW wall $\mathrm{Z}_{\text {eff }}$ decreases from $\approx 2.0$ to 1.2 confirming the dominant impurity for JET-ILW plasmas is beryllium as opposed to carbon [Bresinsek 2013]. Furthermore with the introduction of nitrogen for JET-ILW plasmas $Z_{\text {eff }}$ increases from $\approx 1.2$ up to 1.8 [Giroud 2013, Maddison 2014]. The EPED1 predictions presented in [Leyland 2013] and the most recent EPED1 runs for the low triangularity JET-ILW plasmas presented in this study use bremsstrahlung line-integral measurements of $\mathrm{Z}_{\text {eff. }}$ The EPED1 runs for high triangularity JET-ILW plasmas as presented in [Beurskens $2014]$ and in this study use a constant $Z_{\text {eff }}$ of 2.0. 
The PB stability sensitivity to $\mathrm{Z}_{\text {eff }}$ for JET-ILW plasmas is addressed by [SaarelmaHMWS-2013]. However, further work is required, particularly for the high triangularity plasmas, to address the role of $\mathrm{Z}_{\mathrm{eff}}$ on EPED predictions. 
4. Deuterium fuelling and nitrogen seeding scans in low triangularity JET-ILW plasmas

\subsection{JET-ILW $\mathrm{D}_{2}$ fuelling scan in vertical target, low triangularity configuration}

Figure 3(a) and 3(b) show that for low triangularity VT JET-ILW plasmas there is no significant change in global performance as the confinement enhancement factor $\left(\mathrm{H}_{98}\right)$ and stored thermal energy $\left(\mathrm{W}_{\mathrm{th}}\right)$ remain constant $\left(\mathrm{H}_{98} \approx 0.7\right.$ and $\left.\mathrm{W}_{\text {th }} \approx 4.0 \mathrm{MJ}\right)$ across a deuterium fuelling scan from 0.6 to $4.3 \times 10^{22} \mathrm{el} / \mathrm{s}$. Figure $3(\mathrm{c})$ shows there is no change in the pedestal stored energy with increasing fuelling. Consequently the ratio of the stored thermal energy to the pedestal stored energy is constant as shown by Figure $3(d)$.

Figure 4 presents the measurements of the pressure pedestal structure. Figure 4(a) shows an initial widening of the pedestal at low fuelling, however at higher fuelling there is no significant change. Figure 4(b) shows the peak pressure gradient decreases with increasing deuterium fuelling saturating at higher fuelling. The resulting total pressure pedestal height is constant across the deuterium scan as shown by Figure 4(c). The total pedestal pressure is calculated taking into account ion dilution and is presented to allow a direct comparison to EPED1. The measured average line-integral $\mathrm{Z}_{\mathrm{eff}}$, as provided as an input to EPED1, is near constant, from $\approx 1.3$ to 1.2 , with increasing deuterium fuelling. The EPED1 pedestal width (Figure 4(a)) and height (Figure 4(c)) predictions are constant with increasing fuelling and therefore in good agreement with measurements considering the experimental and model $(+/-20 \%)$ uncertainties. 

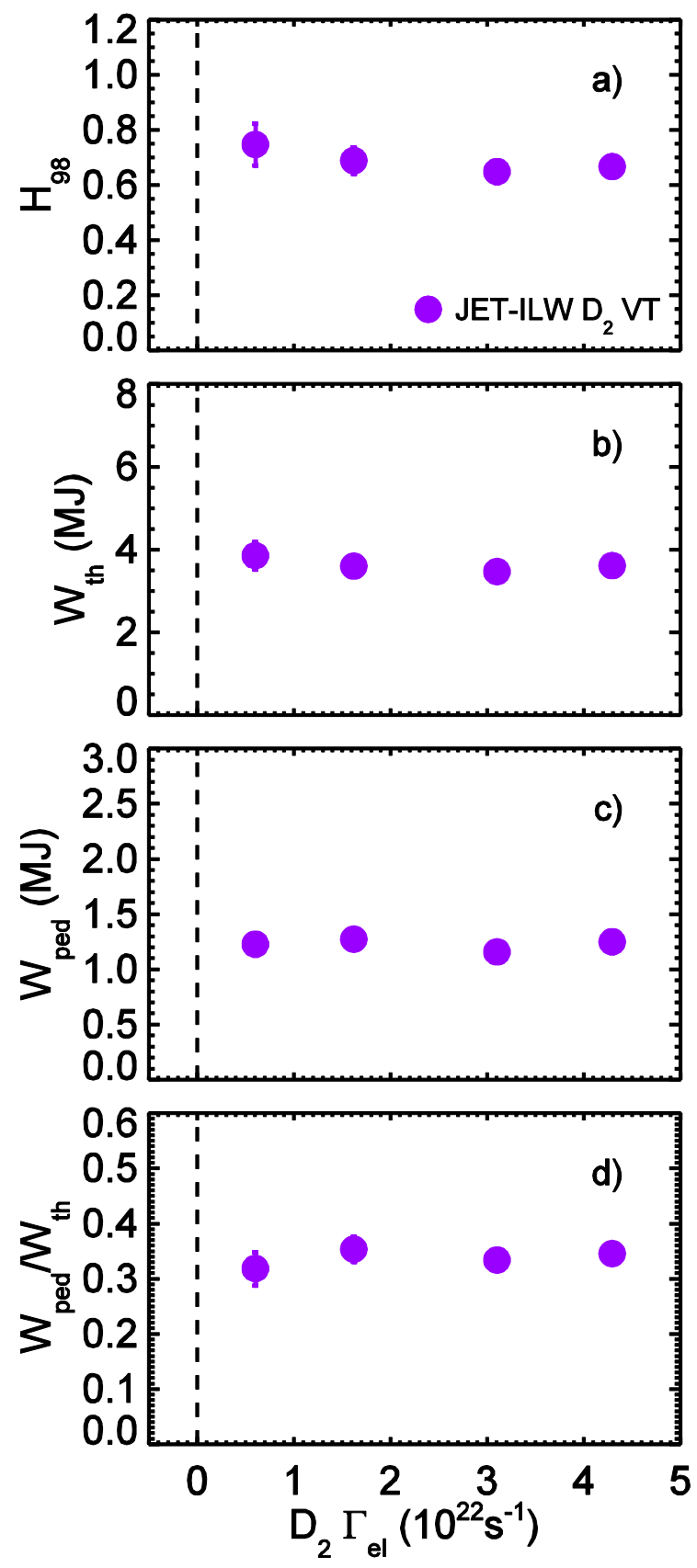

Figure 3. Variation of global and edge performance for low triangularity vertical target JET-ILW plasmas across a deuterium fuelling scan. (a) Confinement enhancement factor $\left(H_{98}\right),(b)$ total stored thermal energy $\left(W_{t h}\right),(c)$ pedestal stored energy $\left(W_{\text {ped }}\right)$ and $(d)$ as a function of deuterium fuelling. The JET pulse numbers in order of increasing $D_{2}$ fuelling are $83491\left(Z_{\text {eff }} \approx 1.3\right), 83490\left(Z_{\text {eff }} \approx 1.3\right), 83488\left(Z_{\text {eff }} \approx\right.$ $1.2)$ and $83487\left(Z_{\text {eff }} \approx 1.2\right)$. 

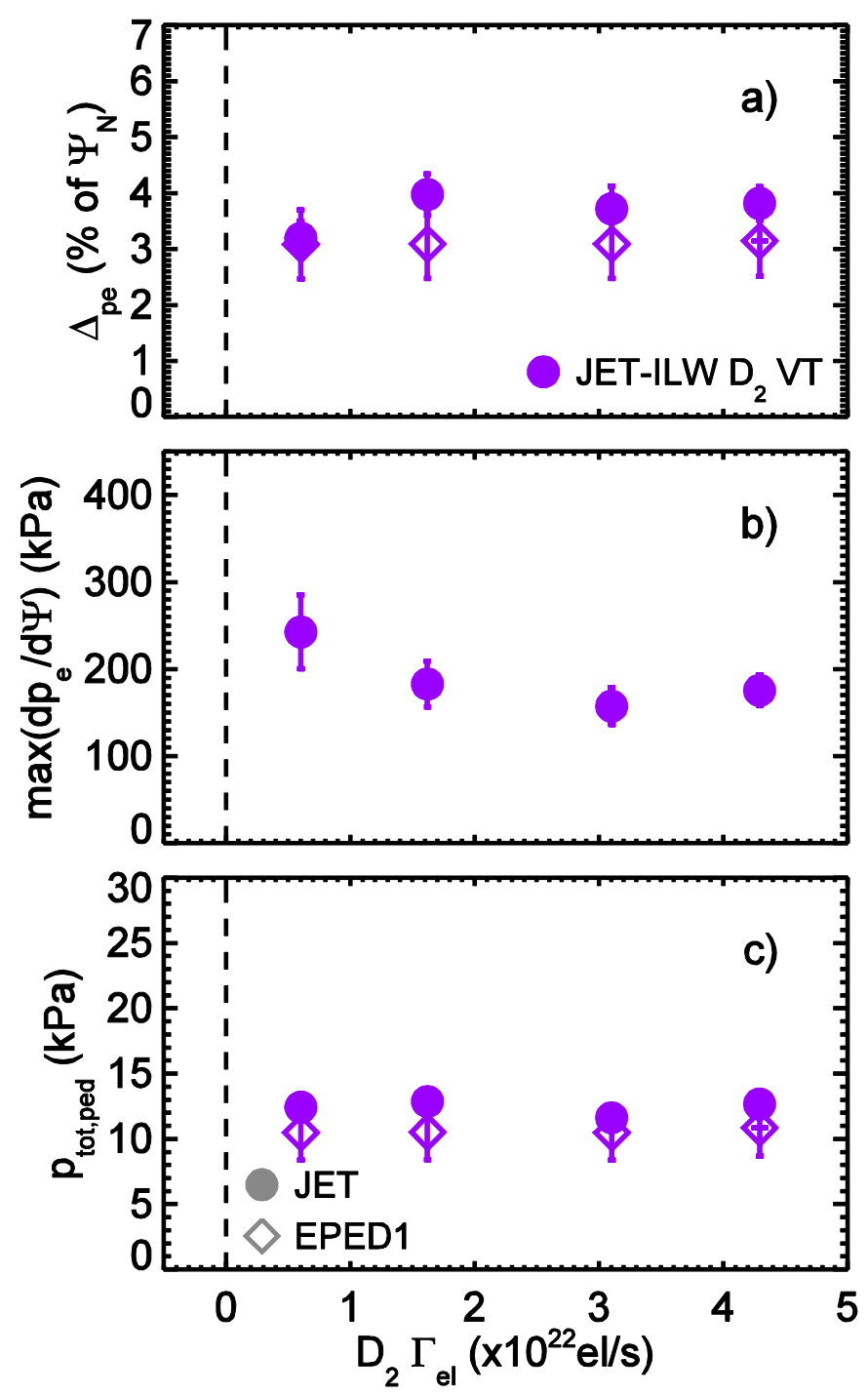

Figure 4. Variation in JET measurements of the pressure pedestal structure (circles) and comparison to EPED1 predictions (diamonds) for low triangularity vertical target JET-ILW plasmas across the same deuterium fuelling scan as shown in Figure

3. (a) Measured pressure pedestal width $\left(\Delta_{p e}\right)$ with corresponding EPED1 predictions, $(b)$ peak pressure gradient $\left(\max \left(d p_{e} / d r\right)\right)$ and $(c)$ total pressure pedestal height ( $\left.p_{\text {tot,ped }}\right)$ as a function of deuterium fuelling with corresponding EPED1 predictions. EPED1 runs incorporate average line-integral measurement of $Z_{\text {eff. }}$ 
Figure 5(a) and 5(b) shows across a nitrogen seeding scan for low triangularity horizontal target JET-ILW plasmas there is no significant change in $\mathrm{H}_{98}$ and $\mathrm{W}_{\text {th }}$ with increasing nitrogen seeding $\left(\mathrm{H}_{98} \approx 0.7\right.$ and $\left.\mathrm{W}_{\text {th }} \approx 4.0 \mathrm{MJ}\right)$. Furthermore, Figure 5(c) shows there is no variation in the pedestal stored energy with increasing nitrogen seeding and Figure 5(d) shows the ratio of pedestal stored energy to total stored energy is also unchanged.

Figure 6 presents the pressure pedestal measurements and EPED1 predictions across the nitrogen seeding scan. Figure 6(a) shows the pedestal width is constant with increasing nitrogen seeding. This is the only scan within the study to clearly show that the pedestal width does not increase with increasing gas dosing (deuterium fuelling or nitrogen seeding). Furthermore, the peak pressure gradient (Figure 6(b)) and pressure pedestal height (Figure 6(c)) show no significant change with increasing nitrogen seeding. The measured line-integral $\mathrm{Z}_{\mathrm{eff}}$ is modified by the introduction of nitrogen as increases from $\approx 1.2$ to 1.5 , as incorporated into the EPED1 predictions. The EPED1 width (Figure 6(a)) and height (Figure 6(c)) predictions are constant with increasing nitrogen seeding and both are in good agreement with the pedestal measurements. It is noted that the EPED1 width predictions are consistently 11 to $16 \%$ lower however this is within the model uncertainty of $+/-20 \%$.

To summarise, the low triangularity JET-ILW deuterium fuelling and nitrogen seeding scans presented in this study show no significant change in performance and a minimal change in pedestal structure with gas puff level. Consequently there is also no significant change in the normalised poloidal pedestal pressure $\left(\beta_{\text {pol,ped }} \approx 0.18\right)$. There is good quantitative agreement with square root relationship, $\Delta \approx 0.076 \sqrt{ } \beta_{\text {pol,ped }}$, within experimental uncertainty however it is difficult to identify any trend across these scans due minimal variation in $\beta_{\text {pol,ped. }}$ These plasmas, taking into account measurement and model uncertainty, are in good agreement with EPED1 predictions. 

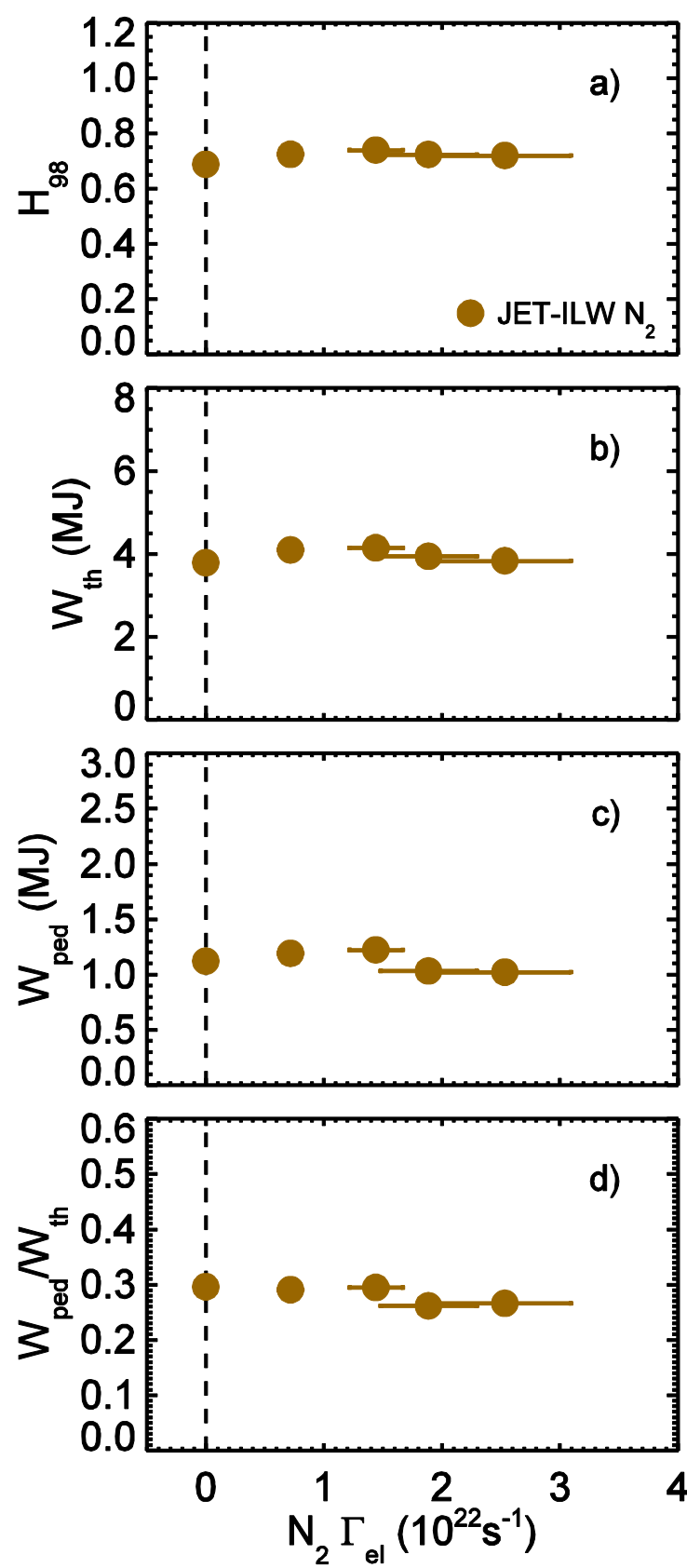

Figure 5. Variation in global and edge performance for low triangularity horizontal target JET-ILW plasmas across a nitrogen seeding scan. The deuterium fuelling level is fixed across the nitrogen seeding scan at $D_{2} \Gamma_{\text {el }} \approx 1.4 \times 10^{22} \mathrm{el} / \mathrm{s}$. (a) Confinement enhancement factor $\left(H_{98}\right)$, (b) total stored thermal energy $\left(W_{t h}\right),(c)$ pedestal stored energy $\left(W_{\text {ped }}\right)$ and $(d) W_{\text {ped }} / W_{\text {th }}$ as a function of nitrogen seeding. The JET pulse numbers in order of increasing $N_{2}$ seeding are $83177\left(Z_{\text {eff }} \approx 1.2\right), 83180\left(Z_{\text {eff }} \approx 1.3\right)$, $83179\left(Z_{\text {eff }} \approx 1.3\right), 83182\left(Z_{\text {eff }} \approx 1.5\right)$ and $83178\left(Z_{\text {eff }} \approx 1.4\right)$. 


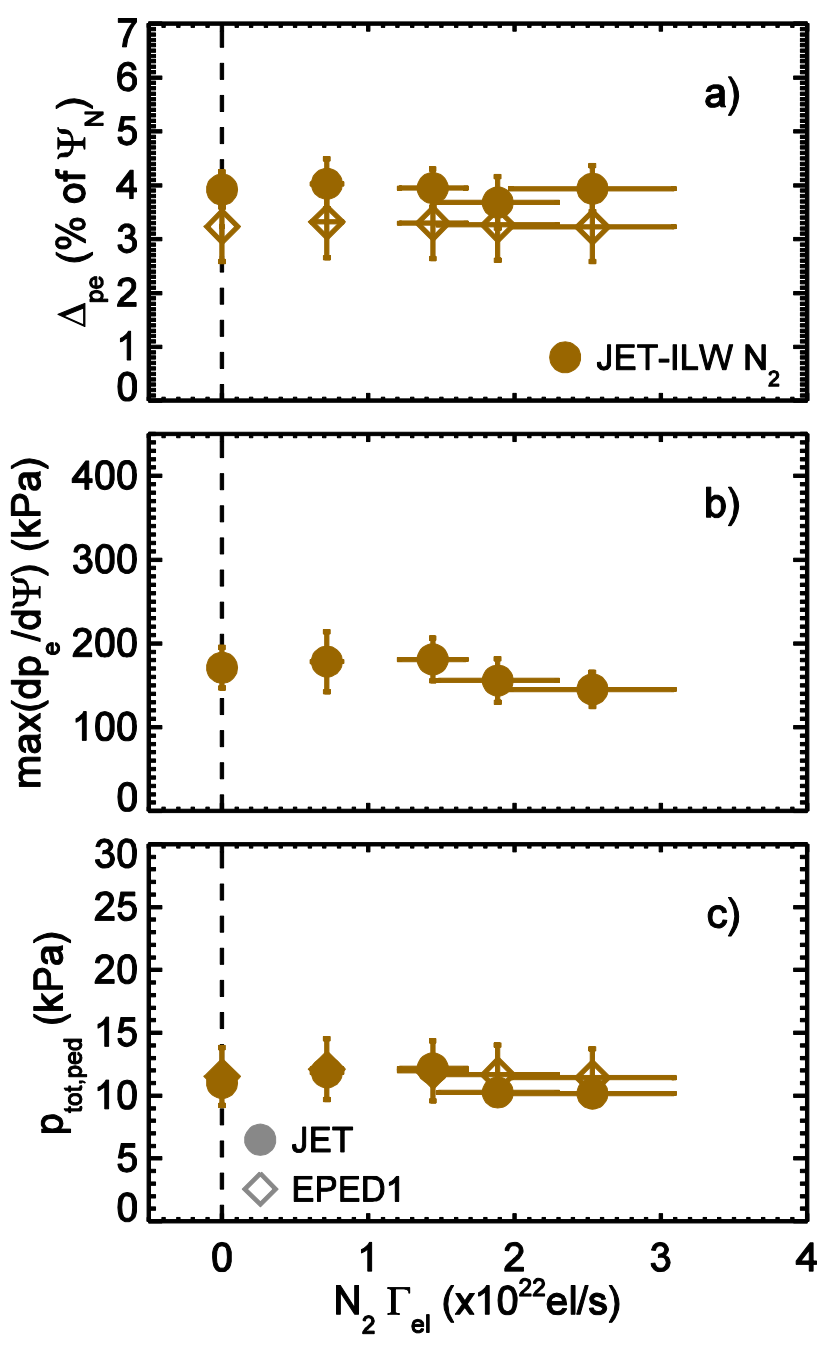

Figure 6. Variation in JET measurements of the pressure pedestal structure (circles) and comparison to EPED1 predictions (diamonds) across a across the same nitrogen seeding scan as shown in Figure 5. (a) Measured pressure pedestal width $\left(\Delta_{p e}\right)$ with corresponding EPED1 predictions, $(b)$ peak pressure gradient $\left(\max \left(d p_{e} / d r\right)\right)$ and $(c)$ total pressure pedestal height $\left(p_{\text {tot,ped }}\right)$ as a function of nitrogen seeding with corresponding EPED1 predictions. EPED1 runs incorporate average line-integral measurements of $Z_{\text {eff. }}$ 
5. Deuterium fuelling and nitrogen seeding scans in high triangularity JET-C and JET-ILW plasmas

\subsection{Plasma performance}

The left and right hand columns of Figure 7 show the performance of high triangularity JET-ILW plasmas with increasing deuterium fuelling and nitrogen seeding respectively. There are two nitrogen scans corresponding to a similar fixed level of deuterium fuelling at $\Gamma_{\mathrm{el}} \approx 1.2 \times 10^{22} \mathrm{el} / \mathrm{s}$ (series 1 ) and $0.8 \times 10^{22} \mathrm{el} / \mathrm{s}$ (series 2).

Figure 7 (a) and 7(c) show there is $\mathrm{a} \approx 20-30 \%$ reduction in global performance for equivalent high triangularity plasmas after the installation of the ILW. $\mathrm{H}_{98} \approx 0.7-0.8$ and $\mathrm{W}_{\text {th }} \approx 3.7-4.5 \mathrm{MJ}$ for JET-ILW deuterium fuelled plasmas in comparison to equivalent JET-C plasmas where $\mathrm{H}_{98} \approx 1.0$ and $\mathrm{W}_{\text {th }} \approx 6.0 \mathrm{MJ}$. Furthermore $\mathrm{H}_{98}$ and $\mathrm{W}_{\text {th }}$ decrease with increasing deuterium fuelling for JET-ILW plasmas unlike the JETC plasmas. As reported in [Leyland 2013] the JET-C plasmas maintain and even improve overall performance $\left(\mathrm{H}_{98}\right.$ and $\left.\mathrm{W}_{\text {th }}\right)$ with increasing deuterium fuelling due to a transition from pure Type I ELMs to the so called mixed Type I/II ELMs [Saibene 2002, Saibene 2005].

The premise behind mixed Type I/II ELMs is there is an increased continuous loss (Type II ELMs) between the large periodic transient collapses of the pedestal (Type I ELMs) prolonging the build up to criticality and consequently decreasing the ELM frequency. This is consistent with measurements presented in [Leyland 2013]. Furthermore, there is a change in magnetic fluctuation behaviour corresponding to washboard modes which are thought to regulate the build-up in pressure by enhanced inter-ELM transport [Perez 2004, Perez 2008].

The behaviour of global performance is linked to the pedestal stored energy ( $\mathrm{W}_{\text {ped }}$ ). Figure $7(\mathrm{e})$ shows $\mathrm{W}_{\text {ped }}(\approx 1.3 \mathrm{MJ})$ does not improve for the JET-ILW plasmas with increasing deuterium fuelling. The edge performance increases relative to the core performance for equivalent JET-C plasmas with increasing deuterium fuelling as $\mathrm{W}_{\text {ped }} / \mathrm{W}_{\text {th }}$ increases from $\approx 0.34$ to 0.40 , see Figure $7(\mathrm{~g})$. However this is not the case for the JET-ILW plasmas as $\mathrm{W}_{\text {ped }} / \mathrm{W}_{\text {th }}$ remains approximately constant $\approx 0.33$

Figure 7(b) and 7(d) shows the global performance initially increases and then saturates $\left(\mathrm{H}_{98} \approx 0.80-0.88\right.$ and $\left.\mathrm{W}_{\text {th }} \approx 4.3-5.3\right)$ for both JET-ILW nitrogen seeding scans. This improvement in performance is mostly due to an increase in $\mathrm{W}_{\text {ped }}$ from $\approx$ 1.4 to $2.0 \mathrm{MJ}$ (see Figure 7(f)). $\mathrm{W}_{\text {ped }}$ initially increases and then saturates like $\mathrm{H}_{98}$ and $\mathrm{W}_{\text {th }}$. Figure $7(\mathrm{~h})$ shows the ratio of $\mathrm{W}_{\text {ped }} / \mathrm{W}_{\text {th }}$ increases and then saturates with increasing nitrogen seeding.

To summarise, the performance behaviour of the JET-ILW high triangularity nitrogen seeded plasmas is akin to the JET-C deuterium fuelled plasmas. This is due to the improvement in overall performance being attributed to the increase in pedestal stored energy at higher gas dosing. In contrast to the JET-C plasmas, the global and edge performance of the JET-ILW plasmas with increasing deuterium fuelling decreases. 

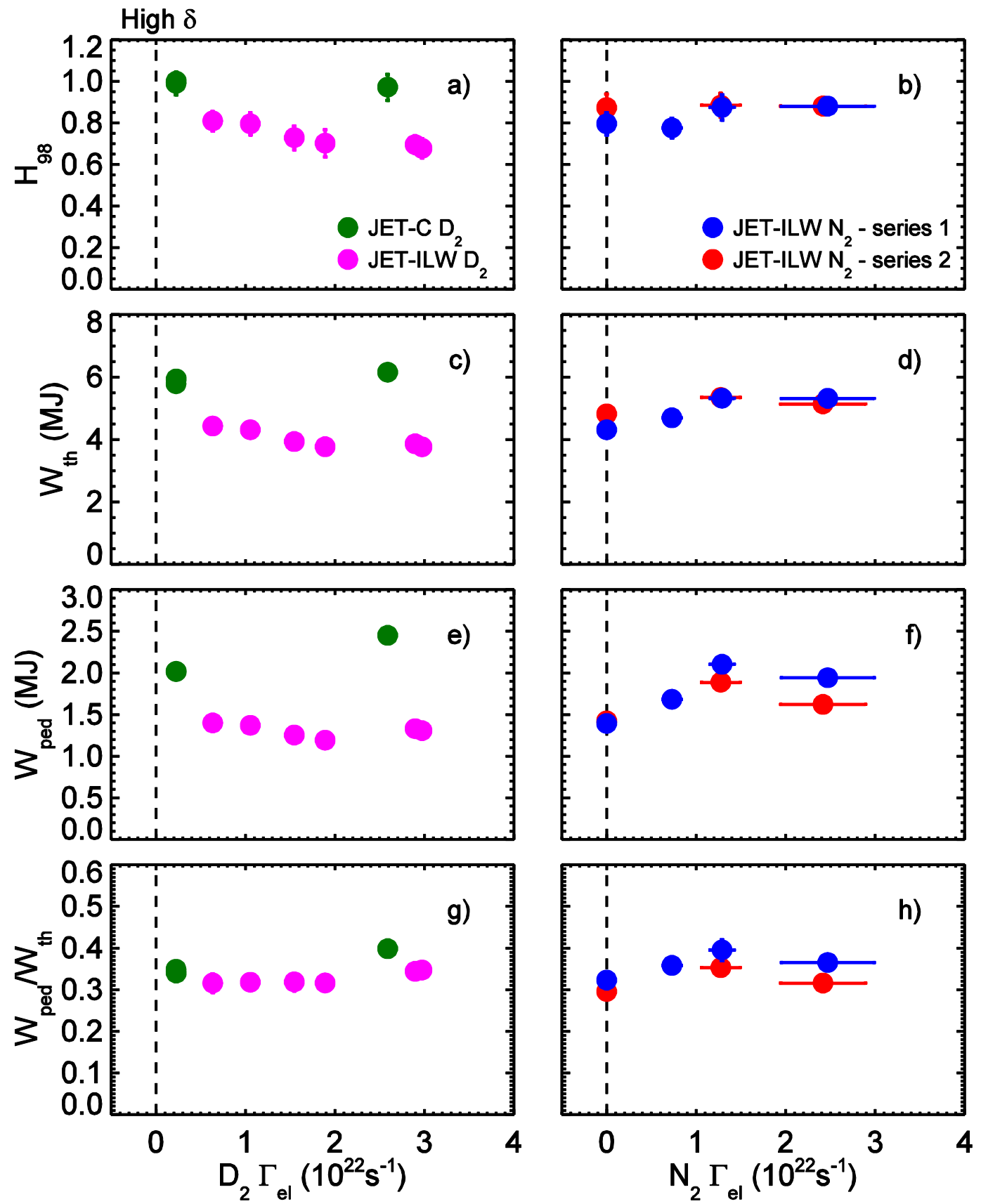

Figure 7. Variation in core and edge performance for a JET-C deuterium fuelling scan (green), a JET-ILW deuterium fuelling scan (pink) and two JET-ILW nitrogen seeding scans (blue and red). The deuterium fuelling level is fixed across the nitrogen seeding scans at $D_{2} \Gamma_{e l} \approx 1.2 \times 10^{22} \mathrm{el} / \mathrm{s}$ for series 1 (blue) and $D_{2} \Gamma_{e l} \approx 0.8 \times 10^{22} \mathrm{el} / \mathrm{s}$ for series $2(\mathrm{red})$. Confinement enhancement factor $\left(H_{98}\right)$ as a function of $($ a) deuterium fuelling and $(b)$ nitrogen seeding. Total stored thermal energy $\left(W_{t h}\right)$ as a function of $(c)$ deuterium fuelling and $(d)$ nitrogen seeding. Pedestal stored energy $\left(W_{\text {ped }}\right)$ as a function of $(e)$ deuterium fuelling and $(f)$ nitrogen seeding. The JET pulse numbers in order of increasing deuterium or nitrogen seeding are as follows. JET-C $D_{2}$ plasmas:

$79498\left(Z_{\text {eff }} \approx 2.0\right), 79499\left(Z_{\text {eff }} \approx 1.9\right)$, and 79503( $\left.Z_{\text {eff }} \approx 1.7\right)$. JET-ILW D $D_{2}$ plasmas; $82586\left(Z_{\text {eff }} \approx 1.3\right), 82585\left(Z_{\text {eff }} \approx 1.3\right), 82541\left(Z_{\text {eff }} \approx 1.3\right), 82540\left(Z_{\text {eff }} \approx 1.3\right), 82806\left(Z_{\text {eff }}\right.$ $\approx 1.3)$ and $82751\left(Z_{\text {eff }} \approx 1.3\right)$. JET-ILW $N_{2}$ series 1 plasmas: $82585\left(Z_{\text {eff }} \approx 1.3\right), 82816$ 
$\left(Z_{\text {eff }} \approx 1.5\right), 82814\left(Z_{\text {eff }} \approx 1.5\right)$ and $82813\left(Z_{\text {eff }} \approx 1.6\right)$. JET-ILW $N_{2}$ series 2: $82588\left(Z_{\text {eff }}\right.$ $\approx 1.3), 82820\left(Z_{\text {eff }} \approx 1.5\right)$ and $82819\left(Z_{\text {eff }} \approx 1.8\right)$. 


\subsection{Pedestal pressure structure and comparison to the EPED1 model}

The left and right hand column of Figure 8 shows the pressure pedestal structure and EPED1 predictions for the high triangularity JET-ILW plasmas shown in Figure 7.

Figure 8(a) and 8(c) show the pressure pedestal widens in real and flux space respectively $\left(\Delta_{\mathrm{pe}} \approx 1.5-2.7 \mathrm{~cm}\right.$ and $2.9-5.0 \%$ of normalised magnetic flux $)$, across JET$\mathrm{C}$ and JET-ILW deuterium scans [Beurskens 2013]. Figure 8(e) shows for these plasmas the peak pressure gradient decreases with increasing deuterium fuelling however this decrease is weaker for JET-C plasmas $\left(\mathrm{dp} / \mathrm{d} \Psi_{\mathrm{N}} \approx 355-280 \mathrm{kPa}\right)$ in comparison to JET-ILW plasmas $\left(\mathrm{dp} / \mathrm{d} \Psi_{\mathrm{N}} \approx 300-170 \mathrm{kPa}\right)$. The combination of pedestal width and gradient define the pressure pedestal height which, as shown by Figure $8(\mathrm{~g})$, increases from $\mathrm{p}_{\text {tot,ped }} \approx 20$ to $24 \mathrm{kPa}$ with increasing deuterium fuelling for JET-C plasmas. However, for JET-ILW deuterium fuelled plasmas the stronger reduction in gradient counteracts the pedestal widening resulting in the pressure remaining constant at $\mathrm{p}_{\text {tot,ped }} \approx 13 \mathrm{kPa}$. EPED1 does not predict the increase in pedestal width (Figure 8(c)) for JET-C and JET-ILW deuterium fuelled plasmas. EPED1 also does not predict the increase in pressure pedestal height for JET-C plasmas as rationalised by [Leyland 2013]. However EPED1 does predict the pressure pedestal height (Figure 8(g)) in the case of the JET-ILW discharges.

Figure 8(b) and 8(d) show the pressure pedestal also widens with increasing nitrogen seeding for JET-ILW plasmas in real and flux space $\left(\Delta_{\mathrm{pe}} \approx 1.7-2.6 \mathrm{~cm}\right.$ and $3.3-5.0 \%$ of normalised magnetic flux). In contrast to the JET-C and JET-ILW deuterium scans, Figure 8(f) shows with increasing nitrogen seeding for JET-ILW plasmas the peak pressure gradient initially increases before saturating. Both the increasing width and gradient act to increase the pressure pedestal height with increasing nitrogen seeding, as shown by Figure 8(h). EPED1 predicts no change in the pressure pedestal width or height within increasing nitrogen seeding where $\mathrm{Z}_{\mathrm{eff}}$ is fixed at 2.0. However the integral-line measurements of $Z_{\text {eff }}$ range from 1.3 to 1.8 with increasing nitrogen seeding and therefore the injection of an impurity is not accounted for in the model.

In summary, on comparison of JET deuterium (JET-C and JET-ILW) and nitrogen (JET-ILW) scans these measurements highlight two key results. First for plasmas with the presence of a carbon like impurity (carbon for deuterium fuelled JET-C plasmas and nitrogen for nitrogen seeded JET-ILW plasmas) there is an improvement in performance with increasing gas dosing due to the pedestal widening and in the case of JET-C plasmas, a weak reduction in the peak pressure gradient whereas for the JET-ILW plasmas, an improvement in the peak pressure gradient. Second, for the JET high triangularity Type I ELMy H-mode scenario it does not follow that the pedestal widening results in an increase in performance, because for deuterium fuelled JET-ILW plasmas the pressure pedestal widens but the pressure pedestal height remains constant. However, for nitrogen seeded JET-ILW plasmas again the pedestal widens and the pressure pedestal height increases. 

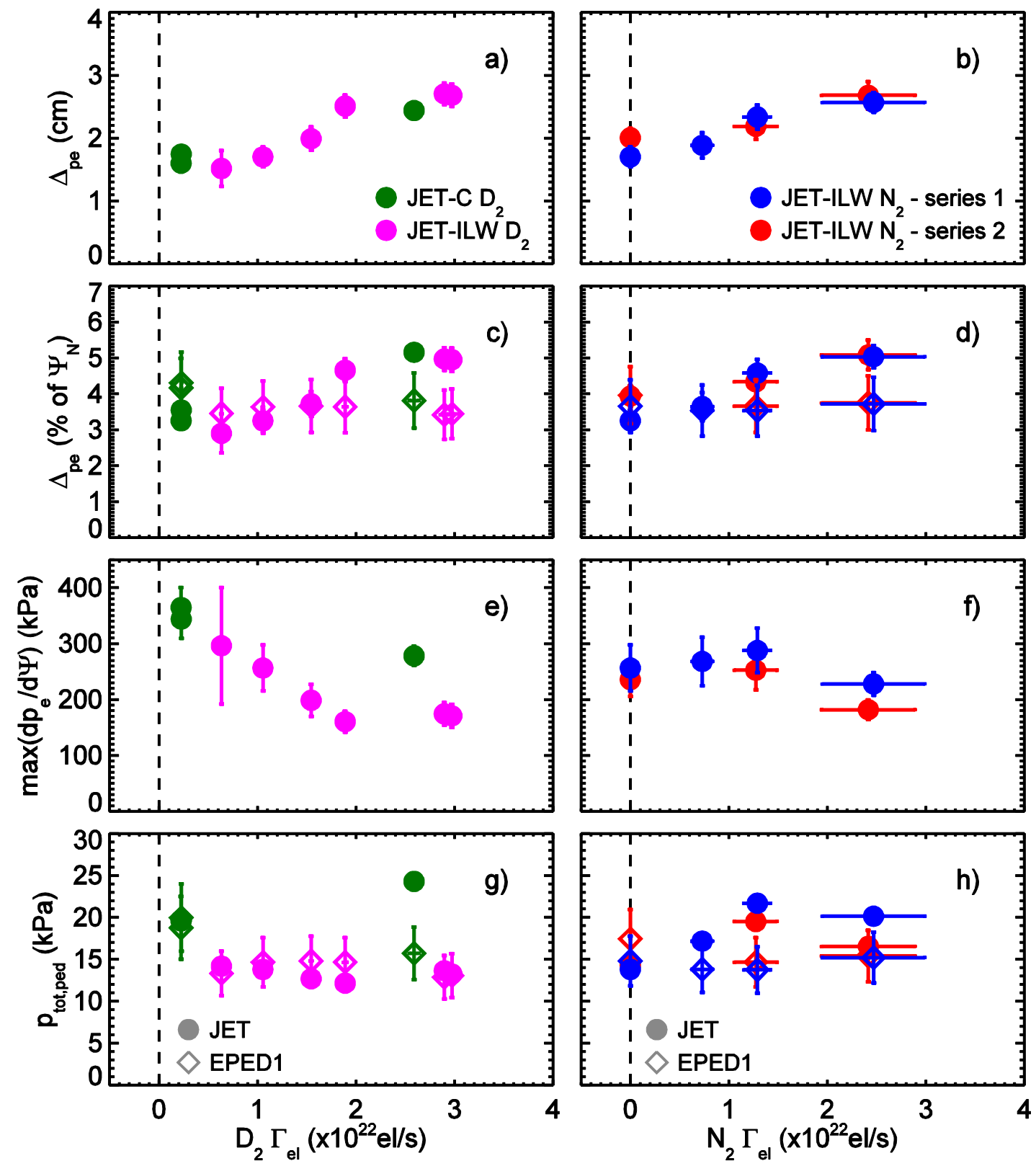

Figure 8. Variation in JET measurements of the pressure pedestal structure (circles) and comparison to EPED1 predictions (diamonds) for the deuterium fuelling and nitrogen seeding scans shown in Figure 7: JET-C deuterium fuelling scan (green), JET-ILW deuterium fuelling scan (pink) and two JET-ILW nitrogen seeding scans (blue and red). Measured pressure pedestal width $\left(\Delta_{p e}\right)$ in $\mathrm{cm}$ as a function of $($ a) deuterium fuelling and $(b)$ nitrogen seeding. Measured pressure pedestal width $\left(\Delta_{p e}\right)$ in percentage of normalised flux and EPED1 predictions as a function of (c) deuterium fuelling and $(d)$ nitrogen seeding. Peak pressure gradient $\left(\max \left(d p_{e} / d r\right)\right)$ as a function of $(e)$ deuterium fuelling and $(f)$ nitrogen seeding. Total pressure pedestal height $\left(p_{\text {tot,ped }}\right)$ as a function of $(g)$ deuterium fuelling and $(h)$ nitrogen seeding. EPED1 runs use fixed value of $Z_{\text {eff }}=2.0$.

Figure 9 shows the measured pedestal width $\left(\Delta_{\mathrm{pe}}\right)$ as a function of measured poloidal pedestal normalised pressure $\left(\beta_{\text {pol,ped }}\right)$ reiterating the latter result, as just discussed, in that the increase in width is not necessarily related to pedestal performance. Figure 9 
also compares measurements with the square root scaling relationship between pedestal width and height which acts as the Kinetic Ballooning constraint in EPED1. The pedestal width increases for the JET-C deuterium scan (green points) with increasing deuterium fuelling and $\sqrt{ } \beta_{\text {pol,ped }}$ however the pedestal width broadening is greater than expected from the $\sqrt{ } \beta_{\text {pol,ped }}$ scaling [Leyland 2013]. The pedestal width for the JET-ILW deuterium scan (pink points) increases with increasing deuterium fuelling. However, unlike JET-C, $\beta_{\text {pol,ped }}$ initially decreases with increasing fuelling resulting in a normal deviation from the $\sqrt{ } \beta_{\text {pol,ped }}$ scaling. Finally, the pedestal width for the two JET-ILW nitrogen scans (red and blue points) increases initially with nitrogen seeding and $\sqrt{ } \beta_{\text {pol,ped }}$ in good agreement with the $\sqrt{ } \beta_{\text {pol,ped }}$ scaling. At the highest $\mathrm{N}_{2}$ seeding rate there is a deviation from the scaling due to the pedestal pressure plateauing. Figure 9 highlights that the deuterium fuelling JET-ILW scan is the most challenging for the EPED1 model as, unlike the deuterium fuelled JET-C and nitrogen seeded JET-ILW plasmas, the deuterium fuelling JET-ILW scan deviates from the scaling relation acting as the kinetic ballooning constraint.

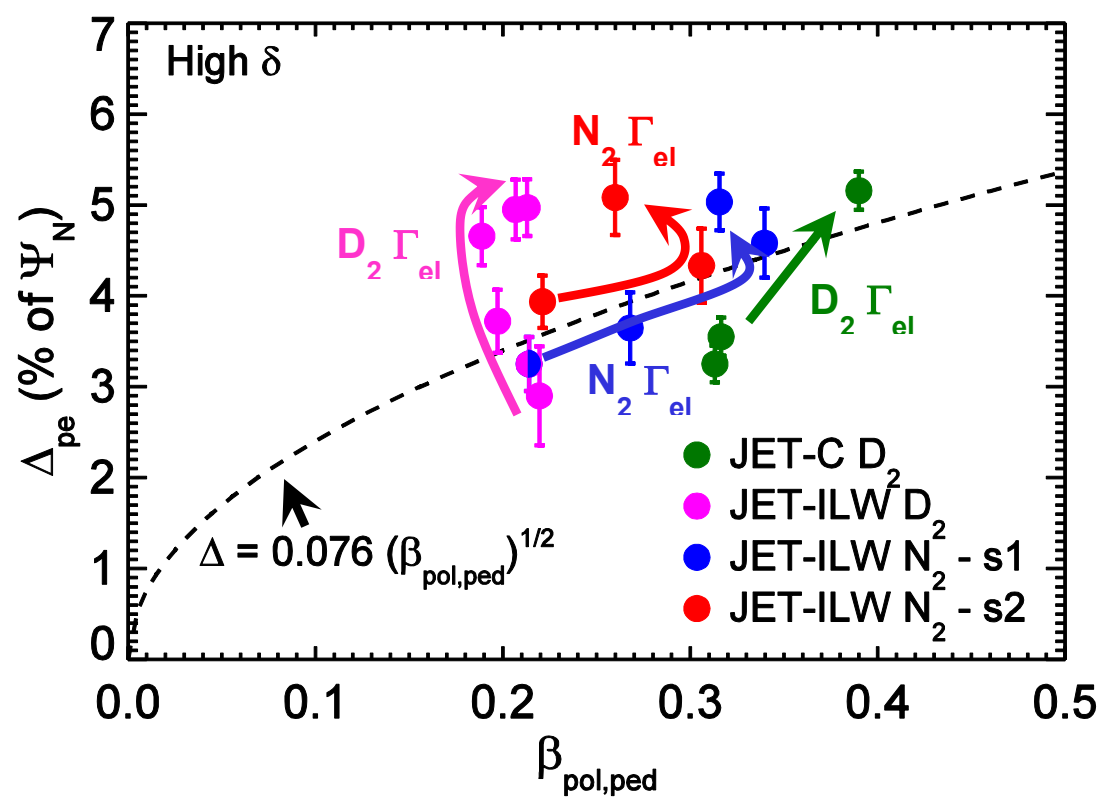

Figure 9. Pressure pedestal width in units of normalised flux $\left(\Delta_{p e}\right)$ as a function of poloidal pedestal normalised pressure $\left(\beta_{\text {pol,ped }}\right)$ for the deuterium fuelling and nitrogen seeding scans shown in Figure 7: JET-C deuterium scan (green solid circles), JET-ILW deuterium scan (pink solid circles) and the two JET-ILW nitrogen seeding scan (solid blue and red circles). The black dashed line shows the square root empirical scaling relationship between the pedestal width $\left(\Delta_{p e}\right)$ and normalised poloidal pedestal pressure $\left(\beta_{\text {pol,ped }}\right)$. 
This section separately examines the variation of the density and temperature pedestal structure. Figure 10 presents the temperature pedestal structure and Figure 11 presents the density pedestal structure. The left and right hand columns show the pedestal structure with increasing deuterium fuelling and nitrogen seeding, respectively, for the scans shown in Figure 7 and 8.

Figure 10(a) and 10(b) show that across both the deuterium fuelling scan (JET-C and JET-ILW plasmas) and the nitrogen seeding scans (JET-ILW plasmas) the temperature pedestal widens, with values ranging from $1.6 \mathrm{~cm}$ at low dosing to $3.2 \mathrm{~cm}$ at high dosing. Figure 10(c) and 10(d) show the peak temperature gradient decreases across both the deuterium fuelling scan and the nitrogen seeding scans. The reduction of the peak temperature gradient is stronger across the deuterium fuelling scan (Figure 10(c)) in comparison to the nitrogen seeding scans (Figure 10(d)). The temperature pedestal height across the deuterium fuelling scan marginally decreases for JET-C plasmas and remains approximately constant for JET-ILW plasmas (Figure 10(e)). In contrast, for nitrogen seeded plasmas, the temperature pedestal height first decreases and then increases with increasing nitrogen seeding (Figure 10(f)). However, overall there is no significant strong change in temperature pedestal height with increasing deuterium fuelling and nitrogen seeding.
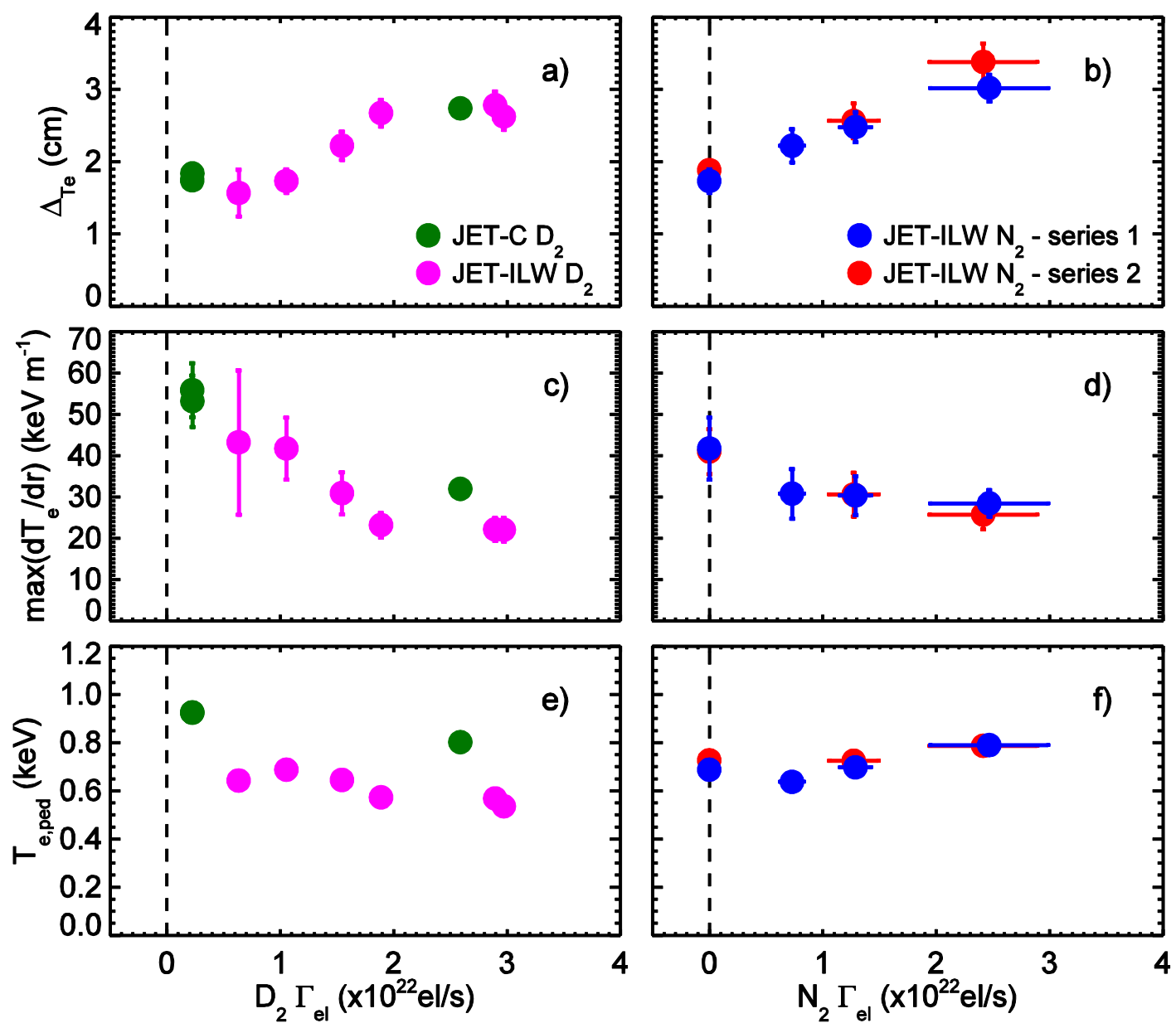
Figure 10. Variation in temperature pedestal structure for the deuterium fuelling and nitrogen seeding scans shown in Figure 7: JET-C deuterium fuelling scan (green), JET-ILW deuterium fuelling scan (pink) and two JET-ILW nitrogen seeding scans (blue and red). Temperature pedestal width $\left(\Delta_{T e}\right)$ as a function of (a) deuterium fuelling and (b) nitrogen seeding. Peak temperature gradient $\left(\max \left(d T_{e} / d r\right)\right)$ as a function of $(c)$ deuterium fuelling and $(d)$ nitrogen seeding. Temperature pedestal height $\left(T_{e, p e d}\right)$ as a function of $(e)$ deuterium fuelling and $(f)$ nitrogen seeding.

Figure 11(a) shows that across JET-C and JET-ILW deuterium scans the density pedestal widens ranging from $1.5 \mathrm{~cm}$ at low fuelling to $2.8 \mathrm{~cm}$ at high fuelling similar to the temperature pedestal width. The peak density gradient, as shown by Figure 11(c), remains constant with increasing deuterium fuelling for JET-C plasmas whereas decreases for JET-ILW plasmas. This variation in peak gradient accounts for the increase in density pedestal height with increasing deuterium fuelling for JET-C plasmas whilst the density pedestal height remains constant for JET-ILW plasmas as shown by Figure 11(e).

The behaviour of the density pedestal width across the two nitrogen scans (JET-ILW) is not as consistent as the temperature pedestal. Figure 11(b) shows the density pedestal width for series 1 (blue) shows no clear trend with increasing nitrogen seeding. However, it is noted that the width does increase from the lowest to highest seeding level. In contrast to series 1 and the deuterium scans the density pedestal width for series 2 shows a weak decrease with increasing nitrogen seeding. The peak density gradient for both nitrogen series initially increases and then saturates as shown by Figure 11(d). Despite the density pedestal width for the nitrogen series 1 not showing a clear trend the pedestal density follows the trend of the peak gradient for both nitrogen series by initially increasing and then saturating, see Figure 11(f). The second pulse $\left(\mathrm{N}_{2} \Gamma_{\mathrm{el}} \approx 0.7 \times 10^{22} \mathrm{el} / \mathrm{s}\right)$ in the blue nitrogen series (JET-ILW $\mathrm{N}_{2}$ - series 1) conforms to the trend in density pedestal height however the pedestal is narrower than the non-seeded pulse and has a relatively high peak gradient.

The key changes in temperature and density pedestal structure which result in a variation of the pressure pedestal and edge performance for JET-C and JET-ILW plasmas are now summarised. First with increasing deuterium fuelling, the pressure pedestal height increases for JET-C plasmas due to a strong increase in the density pedestal height whilst the temperature pedestal height remains constant. Conversely for deuterium fuelled JET-ILW plasmas the pedestal pressure is constant due to the density and temperature pedestal height both remaining constant. The key difference between these plasmas is the variation in the peak density gradient as $\mathrm{dn}_{\mathrm{e}} / \mathrm{dr}$ is constant for JET-C whereas it decreases for JET-ILW. As a result there is not a strong increase in density pedestal height for JET-ILW plasmas as observed for JET-C plasmas.

For nitrogen seeded plasmas the temperature pedestal behaviour is similar to the deuterium fuelled plasmas in that the pedestal broadens and the peak gradient decreases. The key observation for nitrogen seeded plasmas is the change in the density pedestal behaviour. The density pedestal shows no monotomous change in width, however the strong increase in peak gradient results in an increase in density pedestal height. The increase in pressure pedestal width is due to the temperature pedestal widening whilst the density pedestal shows no significant change. This 
increase in pressure pedestal height is due to a strong increase in density pedestal height as well as a relatively small increase in temperature pedestal height.

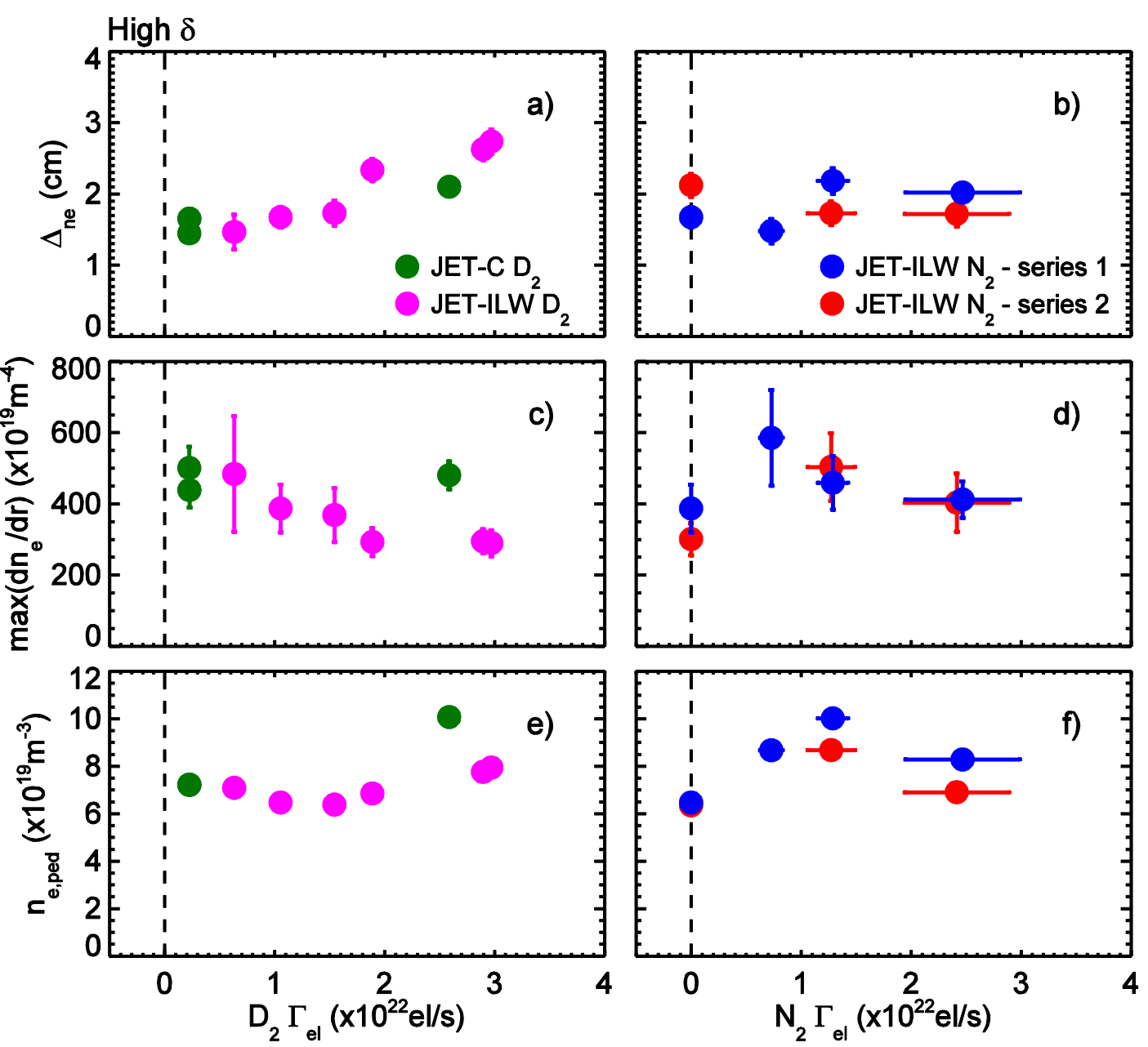

Figure 11. Variation in density pedestal structure for the deuterium fuelling and nitrogen seeding scans shown in Figure 7: JET-C deuterium fuelling scan (green), JET-ILW deuterium fuelling scan (pink) and two JET-ILW nitrogen seeding scans (blue and red). Density pedestal width $\left(\Delta_{n e}\right)$ as a function of $(a)$ deuterium fuelling and $(b)$ nitrogen seeding. Peak density gradient (max $\left.\left(d n_{e} / d r\right)\right)$ as a function of (c) deuterium fuelling and $(d)$ nitrogen seeding. Density pedestal height $\left(n_{e, p e d}\right)$ as a function of $(e)$ deuterium fuelling and $(f)$ nitrogen seeding. 


\subsection{Comparison of EPED1 predictions to larger JET database}

Throughout this study deuterium fuelling and nitrogen seeding scans, typically consisting of 3-6 carefully selected pulses, have been considered. The premise behind focusing on these scans is so any variations in, for example, machine parameters (e.g. use of different gas injection modules) or a variation in machine conditioning between experiments is mitigated as much as possible to improve the clarity of the trends in performance and pedestal structure. However it is essential to consider these scans in the context of a wider set of pulses to provide further confidence in the conclusions. This is particularly important for the comparison of measurements to EPED1 predictions for the high triangularity plasmas where the largest discrepancies are observed.

Figure 12 presents the measured pressure pedestal height and width as a function of the equivalent EPED1 prediction for high triangularity deuterium fuelled JET-C plasmas (triangles), deuterium fuelled JET-ILW plasmas (diamonds) and nitrogen seeded JET-ILW plasmas (circles). Figure 12(a) and 12(b) detail a larger database of JET plasmas whilst Figure 12(c) and 12(d) demonstrate where the scans presented in this paper overlay in the context of this larger dataset. The dashed lines shown in Figure 12 indicate where the measurement is equal to EPED1 and the dotted dashed lines indicate the extremity of EPED1 predictive accuracy $( \pm 20 \%)$.

a)

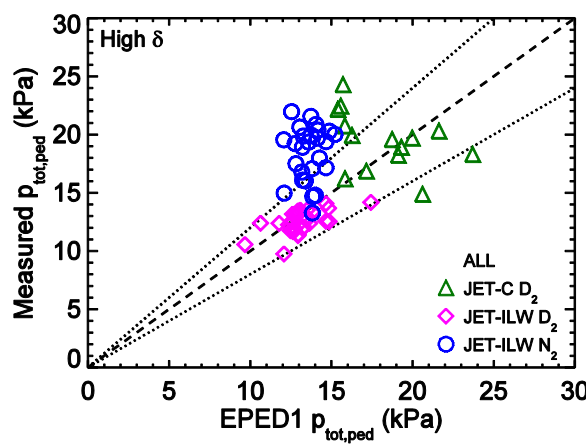

c)

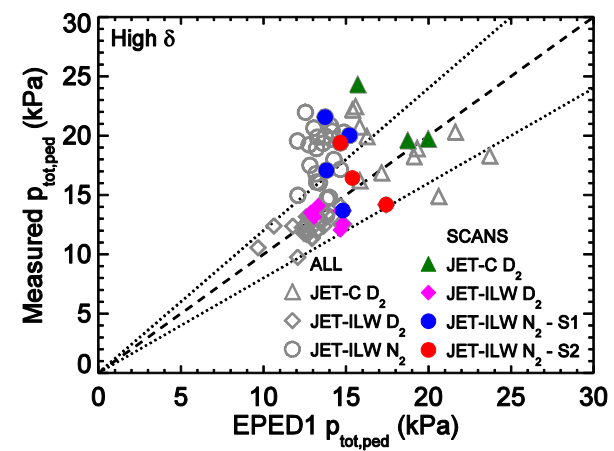

b)

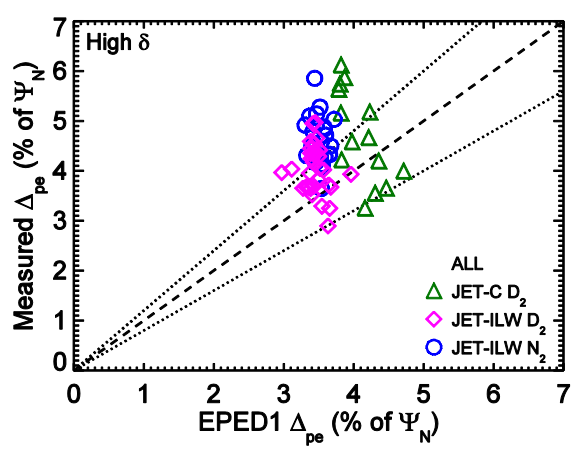

d)

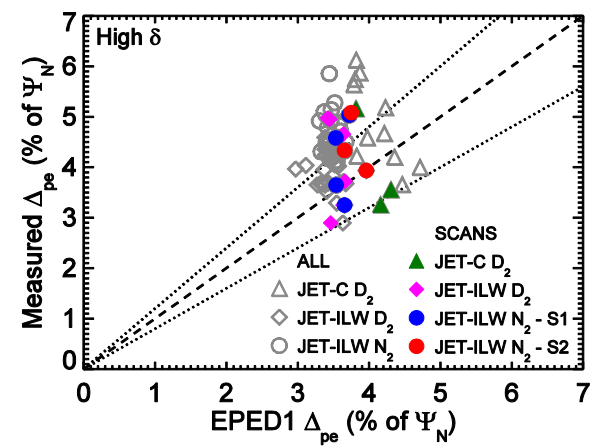

Figure 12. (a), (c) Measured pedestal pressure as a function of EPED1 prediction for pedestal pressure and $(b),(d)$ measured pedestal width as a function of EPED1 prediction for pedestal width all for high triangularity JET-C $D_{2}$ fuelled plasmas (triangles), JET-ILW $D_{2}$ fuelled plasmas (diamonds) and JET-ILW $N_{2}$ nitrogen seeded plasmas (circles). (a), (b) Larger database of JET plasmas for 15 JET-C D $D_{2}$ (green), 36 JET-ILW $D_{2}$ (magenta) and $30 \mathrm{JET}-I L W \mathrm{~N}_{2}$ (blue) plasmas. (c), (d) Comparison of larger database (open grey) to fuelling and seeding scans (closed coloured symbols) as presented throughout this study. The dashed line indicates where 
measurement is equal to the EPEDI prediction and the dotted dashed lines indicate accuracy of the EPED1 predictions, $\pm 20 \%$. EPED1 runs use fixed value of $Z_{\text {eff }}=2.0$.

Figure 12(a) compares the pressure pedestal height measurements and EPED1 predictions. There is a larger scatter for the JET-C $\mathrm{D}_{2}$ fuelled plasmas, see [Leyland 2013], where a systematic deviation is found for the highest deuterium fuelling levels. The cluster of deuterium fuelled JET-ILW plasmas are centred on the black dashed line indicating good agreement between the JET measurements and EPED1. However the cluster of nitrogen seeded JET-ILW plasmas is centred along the upper dotted line at the extremity of the EPED1 accuracy. EPED1 under predicts the pressure pedestal height relative to the measurement as shown in detail by Figure 8 when considering the nitrogen scans.

EPED1 is a combined height and width model and therefore it is important to consider the width as shown in Figure 12(b) along with Figure 12(a). Figure 12(b) demonstrates that for high triangularity plasmas the measurements all show a significant range of pedestal widths corresponding to an increase in deuterium fuelling or nitrogen seeding as concluded from the scans. This is not captured by the EPED1 predictions as reflected by a vertical scatter of each group of pulses (deuterium fuelled JET-C plasmas, deuterium fuelled JET-ILW plasmas and nitrogen seeded JET-ILW plasmas) with points spanning the full extent of the EPED1 predictive accuracy and above. This implies the square root relationship between width $(\Delta)$ and normalised pressure $\left(\beta_{\text {pol,ped }}\right)$ is not always applicable for JET plasmas and may depend on factors such as the density regime, impurity content $\left(Z_{\text {eff }}\right)$, position on PB stability diagram and ELM type.

Figure 12(c) and 12(d) show the scans detailed throughout this study (coloured symbols) overlaid onto the larger dataset (grey symbols). Each scan follows the larger cluster of equivalent plasmas demonstrating that the scans reflect the trends observed in the larger database comparison. 


\section{Summary and conclusions}

This paper reports on the JET pedestal structure with the new ITER-Like-Wall, comparing both high and low triangularity plasma performance with equivalent JET carbon wall plasmas. Furthermore there is a comparison of the pedestal measurements to EPED1 predictions.

Low triangularity JET-ILW plasmas in both vertical and horizontal target strike point configurations show no significant change in performance $\left(\mathrm{H}_{98}, \mathrm{~W}_{\mathrm{th}}, \mathrm{W}_{\mathrm{ped}}\right)$ and pedestal structure $\left(\Delta_{\mathrm{pe}}, \mathrm{p}_{\mathrm{e} \text {,ped }}\right)$ with the gas puff level and these results are in good agreement with EPED1 predictions.

For the high triangularity discharges, deuterium fuelling and nitrogen seeding scans with the ITER-like wall have revealed a number of new features in pedestal behaviour on JET. Pure deuterium fuelled discharges with the JET-ILW show an overall 20-30\% reduction in performance when compared with equivalent JET carbon wall discharges and, furthermore, it is observed that there is no improvement in performance with increasing deuterium fuelling levels, in contrast with the carbon wall. HRTS data from these fuelling scans reveals an apparent increase in the pedestal width as the deuterium fuelling levels are increased but, crucially, this increase in pedestal width appears not to be consistent with previous scaling observations (e.g. $\Delta=0.076 \sqrt{ } \beta_{\text {pol,ped }}$ scaling [Snyder 2009]) as the overall pedestal performance does not correspondingly increase. This result has important implications for the physics constraints that determine the pedestal width (for example KBM constraints within EPED models) and is a topic that will require further study in future work.

In contrast to the deuterium fuelled plasmas, the addition of nitrogen seeding to JETILW plasmas does show an increase in the pressure pedestal height, restoring performance to the levels previously observed with the JET carbon wall.

Measurements of the electron temperature and density pedestal structure for these pulses reveal that the reduction in performance for deuterium fuelled plasmas after the installation of the Be/W ILW wall is primarily due to a reduction in the temperature pedestal height. However, the recovery of the pressure pedestal height with increasing nitrogen seeding is predominately due to the increase in density pedestal height whilst the temperature pedestal height also marginally increases. The increase in pressure pedestal height is accompanied by a widening of the pressure pedestal that is primarily attributed to an increase in temperature pedestal width as the corresponding density pedestal width shows no clear trend and the behaviour varies between different scans with increasing nitrogen seeding.

EPED1 predictions for the JET-ILW deuterium fuelled plasmas show very good agreement with the measured pressure pedestal height. However, for the pulses considered here, the observed systematic increase in the pedestal width with increased deuterium fuelling levels (at constant $\beta_{\mathrm{pol}, \mathrm{ped}}$ ) is not predicted. The JET measurements suggest the pressure pedestal formation is not purely governed by the plasma edge as the scrape-off-layer, neutral recycling and wall interactions could all play a role. If this is the case, further work is required to determine how to incorporate these effects into current models. For the JET-ILW nitrogen seeded plasmas, EPED1 predictions appear to underestimate the measured pressure pedestal height and, as with the pure deuterium fuelled discharges, the observed widening of the pedestal pressure is not 
captured by the model. Further work is required to identify the origins (for example, the role of $Z_{\text {eff }}$ profiles) and significance of these discrepancies, as well as further refining and understanding the uncertainties associated with the measurements and the model. 


\section{References}

[Beurskens-PPCF-2009] Beurskens M.N.A., et al., 2009. Pedestal width and ELM size identity studies in JET and DIII-D; implications for ITER. Plasma Phys. Control. Fusion, 51:124051.

[Beurskens 2009] Beurskens M.N.A, et al., 2009. Pedestal and scrape-off layer dynamics in ELMy H-mode plasmas in JET. Nuclear Fusion, 49:125006.

[Beurskens 2011] Beurskens M.N.A., et al., 2011. H-mode pedestal scaling in DIII-D, ASDEX Upgrade, and JET. Physics of Plasmas, 18:056120.

[Beurskens 2013] Beurskens M.N.A., et al., 2013. The effect of a metal wall on confinement in JET and ASDEX Upgrade. Plasma Phys. Control. Fusion, 55:124043.

[Beurskens 2014] Beurskens M.N.A., et al., 2014. Global and pedestal confinement in JET with a Be/W metallic wall. Nucl. Fusion, 54:043001.

[Connor 1998] Connor J.W. et al., 1998. Magnetohydrodynamic stability of tokamak edge plasmas. Physics of Plamsas, 5:2687.

[Frassinetti 2012] Frassinetti L. et al., 2012. Spatial resolution of the JET Thomson scatering system. Rev. Sci. Instrum., 83:082001.

[Giroud 2012] Giroud C., et al., 2012. Integraion of a radiative divertor for heat load control into JET high triangularity ELMy H-mode plasmas. Nuclear Fusion, 52:063022.

[Giroud-IAEA-2012] Giroud C., et al., 2012. IAEA

[Giroud 2013] Giroud C., et al., 2013. Impact of nitrogen seeding on confinement and power load control of a high-triangularity JET ELMy H-mode plasma with a metal wall. Nucl. Fusion, 53:113025.

[Groebner 1998] Groebner R.J. and Carlstrom T.N., 1998. Critical edge parameters for H-mode transition in DIII-D. Plasma Phys. Control. Fusion, 40:673-677.

[IPB-C2-1999] ITER physics basis expert groups on confinement and transport and confinement modelling and database, ITER physics basis editors: Chapter 2. Plasma confinement and transport. 1999. Nuclear Fusion, 39:2175.

[IPB-C2 2007] ITER Physics Basis, 2007. Chapter 2: Plasma confinement and transport. Nuclear Fusion, 47:S18-S127.

[ITER H-mode database working group 1994] ITER H-mode database working group, 1994. ITER H mode confinement database update. Nuclear Fusion, 34:131.

[Joffrin 2014] Joffrin E., et al., 2014. First scenario development with the JET new ITER-like wall. Nuclear Fusion, 54:013011. 
[Leyland 2013] Leyland M.J., et al., 2013. Pedestal study across a deuterium fuelling scan for high $\delta$ ELMy H-mode plasmas on JET with the carbon wall. Nuclear Fusion, 53:083028

[Maddison 2011] Maddison G.P., et al., 2011. Moderation of divertor heat loads by fuelling and impurity seeding in well-confined ELMy H-mode plasmas on JET. Nuclear Fusion, 51:042001.

[Maddison 2014] Maddison G.P., et al., 2014. Contrasting H-mode behaviour with fuelling and nitrogen seeding in the all-carbon and metallic version of JET. Nuclear Fusion, 54:073016.

[Matthews 2011] Matthews G.F., et al., 2011. JET ITER-like wall-overview and experimental programme. Phys. Scr., T145:014001.

[Morgan 1985] Morgan P.D., et al., 1985. Spectroscopic measurements on the Joint European Torus using optical fibres to relay visible radiation. Rev. Sci. Instrum. 56:862.

[Pasqualotto 2004] Pasqualotto R., et al., 2004. High resolution Thomson scattering for joint european torus (JET). 15th Topical Conf. on High-Temperature Plasmas Diagnostics (San Diego, CA, 2004); Rev. Sci. Instrum., 75:3891-3.

[Perez 2004] Perez C.P., et al., 2004. Washboard modes as ELM-related events in JET. Plasma Physics and Controlled Fusion, 46:61-87.

[Perez 2008] Perez C.P., et al. , 2008. Identifying the MHD signature and power deposition characteristics associated with type-II ELMs in ASDEX Upgrade, Plasma Physics and Controlled Fusion, vol. 50, 065018.

[Philipps 2010] Philipps V., et al., 2010. Overview of the JET ITER-like Wall Project. Fusion Engineering and Design, 85:1581-1586.

[Pitts 2009] Pitts R.A., et al., 2009. Status and physics basis of the ITER divertor. Phys. Scr., T138:014001.

[Ryter 2001] Ryter F., et al., 2001. Experimental Evidence for Gradient LengthDriven Electron Transport in Tokamaks. Phys. Rev. Lett., 86:2325-2328.

[Saarelma-HMWS-2013] Saarelma S., et al., 2013. Global Ideal MHD and Gyrokinetic Pedestal Stability of JET with a Carbon and Metal wall and a Comparison with MAST H-mode Plasmas. Proc. $14^{\text {th }}$ International Workshop on Hmode Physics and Transports Barriers (Fukuoka, Japan, 2013)

[Saibene 2002] Saibene G., et al., 2002. Improved performance of ELMy H-modes at high density by plasma shaping in JET. Plasma Physics and Controlled Fusion, 44:1769. 
[Saibene 2005] Saibene G. et al., 2005. Characterization of small ELM experiments in highly shaped single null and quasi-double-null plasmas in JET. Nucl. Fusion, 45:297-317.

[Scannell 2011] Scannell R. et al., 2011. Deconvolution of Thomson scattering temperature profiles. Rev. Sci. Instrum., 85:053501.

[Snyder 2009] Snyder P.B., et al., et al., 2009. Development and validation of a predictive model for the pedestal height. Phys. Plasmas, 16:056118.

[Snyder 2011] Snyder P.B., et al., 2011. A first-principles predictive model of the pedestal height and width: development, testing and ITER optimization with the EPED model. Nucl. Fusion, 51:103016. 